\title{
Long-Term Measures of Dyslipidemia, Inflammation, and Oxidative Stress in Rats Fed a High-Fat/High-Fructose Diet
}

\author{
Christine Feillet-Coudray $^{1}$ - Gilles Fouret ${ }^{1} \cdot$ Claire Vigor $^{2} \cdot$ Béatrice Bonafos $^{1} \cdot$ Bernard Jover $^{3}$. \\ Agnieszka Blachnio-Zabielska ${ }^{4,5} \cdot$ Jennifer Rieusset $^{6} \cdot$ François Casas $^{1} \cdot$ Sylvie Gaillet $^{1}$ • \\ Jean Francois Landrier ${ }^{7}$ Thierry Durand ${ }^{2} \cdot$ Charles Coudray $^{1}$
}

\begin{abstract}
Inflammation and oxidative stress are thought to be involved in, or associated with, the development of obesity, dyslipidemia, hepatic steatosis, and insulin resistance. This work was designed to determine the evolution of inflammation and oxidative stress during onset and progression of hepatic steatosis and glucose intolerance. Seventy-five male Wistar rats were divided to control and high-fat high-fructose (HFHFr) groups. A subgroup of each group was sacrificed at 4, 8, 12, 16, and 20 weeks. HFHFrfed rats exhibited overweight, glucose intolerance, and
\end{abstract}

Charles Coudray

charles.coudray@inra.fr

1 DMEM (Dynamique Musculaire \& Métabolisme) INRA, University of Montpellier, 2 Place Viala, 34060 Montpellier, France

2 Institut des Biomolécules Max Mousseron, IBMM, University of Montpellier, CNRS, ENSCM, 15 Avenue Charles Flahault, 34090 Montpellier, France

3 PhyMedExp, University of Montpellier, INSERM, CNRS, 371 avenue Doyen Gaston Giraud, 34295 Montpellier, France

4 Physiology Department, Medical University of Bialystok, Jana Kilińskiego 1, 15-089 Bialystok, Poland

5 Epidemiology and Metabolic Disorders Department, Medical University of Bialystok, Jana Kilińskiego 1, 15-089 Bialystok, Poland

6 UMR U1060, INSERM, Faculté de médecine Lyon-Sud, 165 Chemin du Grand Revoyet, 69921 Oullins, France

7 Aix Marseille University, INSERM, INRA, C2VN, 27 boulevard Jean Moulin 13385, Marseille, France hepatic steatosis with increased contents of hepatic diacylglycerols and ceramides. The HFHFr diet increased hepatic interleukin 6 (IL-6) protein and adipose tissue CCL5 gene expression and hepatic nicotinamide adenine dinucleotide phosphate (NADPH) oxidase activity but not mitochondrial reactive oxygen species (ROS) production. The HFHFr diet decreased plasma and liver levels of isoprostanoid metabolites as well as plasma thiobarbituric acid-reactive substance (TBARS) levels. Hepatic glutathione content was decreased with a moderate decrease in superoxide dismutase (SOD) and glutathione peroxidase (GPx) with the HFHFr diet. Overall, HFHFr diet led to hepatic lipid accumulation and glucose intolerance, which were accompanied by only moderate inflammation and oxidative stress. Most of these changes occurred at the same time and as early as 8 or 12 weeks of diet treatment. This implies that oxidative stress may be the result, not the cause, of these metabolic alterations, and suggests that marked hepatic oxidative stress should probably occur at the end of the steatotic stage to result in frank insulin resistance and steatohepatitis. These findings need to be further evaluated in other animal species as well as in human studies.

Keywords Glucose intolerance $\cdot$ Hepatic steatosis $\cdot$ Highfat diet · Inflammation · Isoprostanoids · Lipids · Metabolic syndrome $\cdot$ Obesity $\cdot$ Oxidative stress $\cdot$ Rat

Lipids (2019).

$\begin{array}{ll}\text { Abbreviations } & \\ \text { ADP } & \text { adenine diphosphate } \\ \text { AGE } & \text { advanced glycation end products } \\ \text { ALAT } & \text { alanine aminotransferase } \\ \text { AUC } & \text { area under the curve }\end{array}$

Abbreviations

ADP

ALAT

area under the curve 


\begin{tabular}{ll} 
Cc15 & chemokine (C-C motif) ligand 5 \\
DAG & diacylglycerols \\
FFA & unesterified fatty acids \\
G & glutamate \\
GPx & glutathione peroxidase \\
GSH & glutathione \\
GssG & oxidized glutathione \\
HFHFr & high-fat high-fructose groups \\
IL-6 & interleukin 6 \\
M & Malate \\
Mcp- & monocyte chemoattractant protein 1 \\
1 (CC12) & \\
MetS & metabolic syndrome \\
MUFA & monounsaturated fatty acids \\
NAFLD & nonalcoholic fatty liver diseases \\
OGTT & oral glucose tolerance test \\
PUFA & polyunsaturated fatty acids \\
qPCR & quantitative polymerase chain reaction \\
RLU & relative luminescence unit \\
ROS & reactive oxygen species \\
RT-qPCR & Real-time quantitative polymerase chain \\
& reaction \\
S & Succinate \\
SFA & saturated fatty acids \\
SOD & superoxide dismutase \\
TAG & triacylglycerols \\
TBARS & thiobarbituric acid-reactive substances \\
TNF- $\alpha$ & tumor necrosis factor- $\alpha$ \\
UHPLC/ & Ultra-high performance liquid chromatogra- \\
MS/MS & phy/mass spectrometry/mass spectrometry \\
WB & western blot \\
\hline &
\end{tabular}

\section{Introduction}

Increased consumption of energy-dense foods containing high amounts of animal fats, saturated fatty acids, and fructose is thought to be the major contributor to the current epidemic overweight and obesity worldwide (Dandona et al., 2005; Zivkovic et al., 2007). Abdominal obesity, dyslipidemia, and nonalcoholic fatty liver diseases (NAFLD) are among the major metabolic syndrome (MetS) features, which include also insulin resistance and hypertension (Johnson et al., 2013). When lipid intake exceeds energy expenditure demand, excess lipid is first accumulated in the adipose tissue, which plays a buffer role in the first high-energy exposure. However, when this reservoir is exceeded, the excess lipid can attain other important metabolic tissues, in particular liver and muscle (ectopic lipids) leading to hepatic steatosis, glucose intolerance, and type-II diabetes development. Moreover, most studies agree that it is not the lipid accumulation that causes glucose intolerance, but rather lipid intermediates such as fatty acyl-CoA, diacylglycerols (DAG), and ceramides and particularly the localization, the composition, and the turnover of these intermediates that play an important role in the development of hepatic steatosis, glucose intolerance, and type-II diabetes (Perry et al., 2014; Petersen and Shulman, 2017).

The first stage of NAFLD is the liver lipid accumulation. This later may come from the lipid diet, de novo lipogenesis or adipose lipid release. (Kawano and Cohen, 2013). Low-grade inflammation and oxidative stress are thought to be involved in the initiation of this metabolic disorder (Anderson et al., 2009; Ando and Fujita, 2009). Together with other cellular factors, high inflammation, free radicals, and marked oxidative stress are generally involved in the progression of hepatic steatosis to steatohepatitis, fibrosis, cirrhosis, and hepatocarcinoma (Ucar et al., 2013). Indeed, high reactive oxygen species (ROS) generation may contribute to tissue damage and dysfunction in different ways: (1) it may directly dysregulate redox-sensitive signaling pathways, and (2) may cause oxidative damage to biological structures. The mitochondrial electron transport chain is known to be one of the major producers of superoxide anions, which are precursors to other ROS such as hydrogen peroxide and hydroxyl radicals. The other important physiological system of cellular ROS production is the NADPH oxidase (Valko et al., 2007).

Mitochondria play a central role in cellular energy metabolism by transforming the energy stored in nutrients into adenosine triphosphate through the reduction of oxygen into water through the complexes of the mitochondrial respiratory chain. However, a small proportion of electrons may react directly with $\mathrm{O}_{2}$ at the level of complexes I and III of the respiratory chain, with production of ROS more or less important.

Emerging evidence implicates mitochondrial dysfunction, with a concomitant increase in ROS production, contributing to obesity-induced fatty liver disease (Mantena et al., 2008). The NADPH oxidase complex is another important source of cellular ROS production that generates ROS in response to a wide range of nutritional and nonnutritional stimuli (Bedard and Krause, 2007). Although NADPH oxidase-ROS production is generally under control and involved in specific signaling pathways, high or uncontrolled activation of NADPH oxidase may lead to ROS overproduction and then to oxidative stress under certain conditions (Paik and Brenner, 2011).

Inflammation and oxidative stress are each independently involved in the development and progression of many metabolic syndrome features. The crucial roles of inflammation and oxidative stress in the development of hepatic diseases have been emphasized for decades, where hepatic inflammation and oxidative stress are sustained and participated in this pathological progressive process 
(Fernandez-Sanchez et al., 2011). Indeed, there is growing evidence that inflammation and oxidative stress are closely related, creating a vicious circle that can, for example, aggravate nonalcoholic fatty liver disease into nonalcoholic steatohepatitis and cirrhosis (Li et al., 2016). Low-grade inflammation is a reported characteristic of the obese state, and adipose tissue releases many inflammatory mediators. These include highly active molecules like leptin, resistin, and adiponectin as well as some more classical cytokines. Most likely, proinflammatory cytokines such as tumor necrosis factor- $\alpha$ (TNF- $\alpha)$ and interleukin 1 and 6 (IL-1 and -6) are released by the inflammatory cell infiltrates including monocytes/macrophages, neutrophils, B lymphocytes, T lymphocytes, and others (Gerner et al., 2013). Obese individuals have been reported to have circulating levels of many inflammatory markers higher than lean individuals, and these may play a major role in insulin resistance and other metabolic disorders (Tordjman et al., 2008).

Therefore, it is generally accepted that the development of metabolic syndrome features, in particular obesity, hepatic steatosis, and insulin resistance, is initiated by, or accompanied by inflammation and oxidative stress in liver and adipose tissue (Renaud et al., 2014). Undeniably, one crucial question that comes up is whether inflammation and oxidative stress contribute to, or are simply a consequence of, the two main features of the metabolic syndrome-that is installation and development of fatty liver and glucose intolerance. The present work was designed to determine the evolution of both inflammation and oxidative stress markers during obesity, hepatic steatosis, and glucose intolerance development in a high-fat high-fructose group (HFHFr) diet-fed rats. The novelty of this work is the investigation of a wide range of measured outcomes of oxidative stress, in particular the isoprostanoid metabolites, in a large longitudinal study with several end-points from 4 to 20 weeks diet treatment with a control group at each time point. This approach allows an integrated overview of the physiological time-course evolution of both inflammation and oxidative stress and their impact on the major MetS features such as obesity, hepatic steatosis, and glucose intolerance development.

\section{Materials and Methods}

\section{Animals and Diets}

Seventy five 4-week-old male Wistar rats ( 100 g, Charles River, L'Arbresle, France) were housed ( 2 per cage) under conditions of constant temperature $\left(20-22{ }^{\circ} \mathrm{C}\right)$, humidity (45-50\%), and a standard dark cycle (20.00-08.00 h). At the beginning of the study, the rats were block randomized into 10 groups corresponding to different sacrifice-time points (five groups fed the control diet and five other groups fed the HFHFr diet). This randomization was based on the initial rat body weight. Fasted rats (seven control rats and eight HFHFr rats) were sacrificed after 4, 8, 12, 16, or 20 weeks on their respective diets.

The control diet contains $4 \%$ lipids as soybean oil, and the HFHFr diet contains $35 \%$ lipids ( $1 \%$ soybean oil $+8.5 \%$ lard $+17 \%$ palm oil $+8.5 \%$ cocoa butter) and $25 \%$ fructose (Table 1). The high-fat diet was supplemented with $1 \%$ soybean oil to maintain an adequate intake of linoleic acid and to avoid growth alterations resulting from linoleic acid deficiency. Refined palm oil (VAMOLINE) was purchased from Vandemoortele Lipids and Dough Division, Izegem, Belgium. The lipid fraction of the control diet was composed of $17.4 \%$ saturated fatty acids (SFA), $28.1 \%$ monounsaturated fatty acids (MUFA), and $54.5 \%$ polyunsaturated fatty acids (PUFA), and that of the HFHFr diet was composed of $44.2 \%$ SFA, $43.2 \%$ MUFA, and $12.6 \%$ PUFA (Table 2). In addition to the high level of lipids in the HFHFr diet, the percent of both SFA and MUFA was greatly increased whereas that of PUFA was substantially decreased compared to the control diet. Rats

Table 1 Diet composition ${ }^{\mathrm{a}}(\mathrm{g} / \mathrm{kg})$

\begin{tabular}{|c|c|c|}
\hline Ingredient & Control & HFHFr \\
\hline Casein & 150 & 180 \\
\hline Cornstarch & 456.8 & 75.1 \\
\hline Maltodextrine & 155 & 25 \\
\hline Sucrose & 100 & 0 \\
\hline Fructose & 0 & 250 \\
\hline Soybean oil & 40 & 10 \\
\hline Lard & 0 & 85 \\
\hline Refined palm oil & 0 & 170 \\
\hline Cocoa butter & 0 & 85 \\
\hline Cellulose & 50 & 60 \\
\hline Mineral mix (AIN-93 M) ${ }^{\mathrm{b}}$ & 35 & 42 \\
\hline Vitamin mix (AIN-93 M) & 10 & 12 \\
\hline L-Cystine & 1.8 & 2.2 \\
\hline Choline chloride & 1.4 & 1.7 \\
\hline Total & 1000 & 1000 \\
\hline
\end{tabular}

Refined palm oil (VAMOLINE) was purchased from Vandemoortele Lipids and Dough Division Prins Albertlaan 12, 8870 Izegem, Belgium. HFHFr, high-fat high-fructose.

${ }^{\mathrm{a}}$ Diet composition was based on the AIN-93 M diet formulation.

${ }^{\mathrm{b}}$ Mineral mix AIN 1993 ensures the following mineral levels in the diets (mg/kg): Na, 1020; K, 3600; P, 4000; Ca, 5000; Mg, 500; Zn, 30; Fe, 35; Cu, 6; Mn, 54; Se,0.1; I, 0.2; and Cr, 2.

${ }^{c}$ Vitamin mix AIN 1993 ensures the following mineral levels in the diets $(\mathrm{mg} / \mathrm{kg})$ : thiamine, 6; riboflavine, 6 ; pyridoxine, 7 ; nicotinic acid, 30; calcium pantothenate, 16; folic acid, 2; D-biotin: 0.2; and $(\mu \mathrm{g} / \mathrm{kg})$ cyanocobalamine (vitamin B12), 10; vitamin $\mathrm{K}, 50$; and (IU/kg) vitamin A, 4000; vitamin E, 50; and vitamin D, 1000. 
Table 2 Fatty acid composition of the experimental diets ${ }^{\mathrm{a}}$ (mg/g diet)

\begin{tabular}{|c|c|c|c|c|}
\hline Fatty acids & Control diet (\%) & Control diet (mg/g) & HFHFr diet (\%) & HFHFr diet (mg/g) \\
\hline $14: 0$ & $0.43 \pm 0.05$ & $0.170 .17 \pm 0.02$ & $1.09 \pm 0.16$ & $3.823 .82 \pm 0.56$ \\
\hline $16: 0$ & $11.7 \pm 0.1$ & $4.684 .68 \pm 0.04$ & $30.4 \pm 0.4$ & $106.106 .4 \pm 1.4$ \\
\hline $16: 1 n-7$ & $0.16 \pm 0.01$ & $0.060 .06 \pm 0.00$ & $0.91 \pm 0.03$ & $3.193 .19 \pm 0.11$ \\
\hline $18: 0$ & $4.43 \pm 0.05$ & $1.771 .77 \pm 0.02$ & $12.1 \pm 0.2$ & $42.342 .35 \pm 0.70$ \\
\hline $18: 1 \mathrm{n}-9$ & $26.1 \pm 0.2$ & $10.410 .44 \pm 0.08$ & $40.1 \pm 0.4$ & $140.140 .35 \pm 1.4$ \\
\hline $18: 1 \mathrm{n}-7$ & $1.53 \pm 0.06$ & $0.610 .61 \pm 0.02$ & $1.37 \pm 0.01$ & $4.794 .79 \pm 0.04$ \\
\hline $18: 2 n-6$ & $48.8 \pm 0.3$ & $19.519 .52 \pm 0.12$ & $11.5 \pm 0.1$ & $40.240 .25 \pm 0.35$ \\
\hline $18: 3 n-3$ & $5.58 \pm 0.05$ & $2.232 .23 \pm 0.02$ & $0.71 \pm 0.02$ & $2.492 .49 \pm 0.07$ \\
\hline 20:x & $0.38 \pm 0.01$ & $0.150 .15 \pm 0.00$ & $0.49 \pm 0.02$ & $1.721 .72 \pm 0.07$ \\
\hline $22: x$ & $0.47 \pm 0.01$ & $0.190 .19 \pm 0.00$ & $0.13 \pm 0.01$ & $0.460 .46 \pm 0.03$ \\
\hline $\mathrm{SFA}^{\mathrm{b}}$ & $17.4 \pm 0.1$ & & $44.2 \pm 0.4$ & \\
\hline MUFA $^{\mathrm{b}}$ & $28.1 \pm 0.2$ & $\begin{array}{c}6.966 .96 \pm 0.04 \\
11.211 .24 \pm 0.08\end{array}$ & $43.2 \pm 0.2$ & $\begin{array}{l}154.154 .7 \pm 1.4 \\
151.151 .2 \pm 0.7\end{array}$ \\
\hline PUFA $^{\mathrm{b}}$ & $54.5 \pm 0.4$ & $21.821 .8 \pm 0.16$ & $12.6 \pm 0.2$ & $44.144 .1 \pm 0.7$ \\
\hline unsaturated FA & $82.6 \pm 0.1$ & $33.033 .04 \pm 0.04$ & $55.8 \pm 0.3$ & $195.195 .3 \pm 1.05$ \\
\hline$n-6$ & $48.8 \pm 0.3$ & $19.519 .52 \pm 0.12$ & $11.6 \pm 0.1$ & $40.640 .6 \pm 0.35$ \\
\hline$n-3$ & $5.67 \pm 0.10$ & $2.272 .27 \pm 0.04$ & $1.00 \pm 0.05$ & $3.5 \pm 3.5 \pm 0.18$ \\
\hline$n-6 / n-3$ & $8.65 \pm 0.12$ & $3.463 .46 \pm 0.05$ & $11.7 \pm 0.1$ & $40.940 .9 \pm 0.35$ \\
\hline PUFA/SFA ${ }^{\mathrm{b}}$ & $3.12 \pm 0.04$ & $1.251 .25 \pm 0.02$ & $0.28 \pm 0.01$ & $0.980 .98 \pm 0.04$ \\
\hline
\end{tabular}

HFHFr, high-fat high-fructose.

${ }^{\mathrm{a}}$ Values are based on identifiable peaks.

${ }^{\mathrm{b}}$ MUFA: monounsaturated fatty acids; PUFA: polyunsaturated fatty acids; and SFA: saturated fatty acids.

were given free access to food and tap water throughout the study. The rat body weight was determined weekly and food consumption was determined every 2 or 3 days. Our institution guidelines for the care and use of laboratory animals were observed and all experimental procedures were approved by the local ethical committee in Montpellier, France (Reference CEEA-LR-12002).

\section{Animal Sacrifice and Metabolic Biochemical Analyses}

At $4,8,12,16$, and 20 weeks of diet, eight to $10 \mathrm{~h}$-fasted rats (23 h:00-08 h:00) were anesthetized with pentobarbital (40 mg/kg b.w.) and blood was drawn from the abdominal artery with a heparinized syringe, to avoid blood coagulation during sampling, and transferred to dry and heparinized tubes. $100 \mu \mathrm{L}$ of heparinized blood were mixed with $900 \mu \mathrm{L}$ of metaphosphoric acid $6 \%$ and centrifuged and the supernatant was recovered for blood glutathione (GSH) and oxidized glutathione (GssG) measurement. Blood tubes were centrifuged at $1000 \mathrm{~g}$ for $10 \mathrm{~min}$ at $4{ }^{\circ} \mathrm{C}$, plasma or serum was collected and stored at $-80{ }^{\circ} \mathrm{C}$ until analysis. Liver and visceral fats (retroperitoneal and epididymal depots) were removed, rinsed with $0.9 \% \mathrm{NaCl}$, weighed and frozen in $\mathrm{N}_{2}$, and kept at $-80{ }^{\circ} \mathrm{C}$ until analyses, except a part of liver that was used for the extemporaneous isolation of liver fresh mitochondria.

\section{Liver Histological Analysis}

For microscopic studies, liver samples were fixed in $10 \%$ neutral buffered formalin and embedded in paraffin. Serial tissue sections $(5 \mu \mathrm{m})$ were processed. Hepatic steatosis was evaluated by histological examination after hematoxylin/eosin staining. Liver sections were observed under a Nikon Eclipse TE300 microscope. Images were acquired using the ToupView software driving a ToupCam CCD camera on at least 10 fields (X200) per section. The vesicular (lipids) area was graded from 1 to 4 , where 1 corresponds to $0-25 \%$ of the tissue area presenting fatty vacuoles, $2 \%$ to $25-50 \%, 3 \%$ to $50-75 \%$, and $4 \%$ to $75-100 \%$. For each group, liver samples from six to eight rats were prepared, stained, and examined.

\section{Hepatic DAG and Ceramide Contents}

The liver ceramide content was measured using a UHPLC/MS/MS approach, as previously described with a minor modification (Blachnio-Zabielska et al., 2012). Briefly, the liver samples $(20 \mathrm{mg})$ were pulverized and homogenized in a solution composed of $0.25 \mathrm{M}$ sucrose, $25 \mathrm{mM} \mathrm{KCl}, 50 \mathrm{mM}$ Tris, and $0.5 \mathrm{mM}$ éthylènediaminetétraacétique (EDTA), pH 7.4. Immediately afterward, the internal standards (d17:1/8:0, d17:1/18:0, d17:1/18:1(9Z), 
d17:1/20:0, d17:1/24:0, and d17:1/24:1(15Z) (Avanti Polar Lipids, Alabaster, AL, USA) were added. The mixture was vortexed, sonicated, and centrifuged. The supernatant was transferred to a new tube and pellet was re-extracted. Supernatants were combined and evaporated under nitrogen. The dried sample was reconstituted in $100 \mu \mathrm{L}$ of $\mathrm{LC}$ Solvent A for LC/MS/MS analysis. Quantitative measurement was made using a triple quadrupole mass spectrometer (Agilent 6460, Santa Clara, CA, USA) in positive mode using multiple reaction monitoring. All ceramides were quantified against standard concentration curves.

The liver DAG content was measured using a UHPLC/MS/MS approach, as previously described (Blachnio-Zabielska et al., 2013). Briefly, DAG were extracted together with ceramides. A known amount of internal standard mix (Deuterated DAG Mixture I and Mixture II - Avanti Polar Lipids) was added to each sample. Next, samples were extracted as described above. The following DAG species were quantified: 18:1/18:2, 16:0/18:2, 16:0/16:0, 16:0/18:1, 18:0/20:0, 18:0/18:1, 18:1/18:1, 18:0/ 18:2, and 16:0/18:0 using UHPLC/MS/MS. The DAG content was analyzed by means of a triple quadrupole mass spectrometer using a positive ion electrospray ionization source with multiple reaction monitoring against the standard concentration curves.

\section{Plasma, Liver, and Adipose Tissue Inflammatory Markers}

The plasma levels of TNF- $\alpha$, IL- 6 , and MCP- 1 were measured using cytokine-specific enzyme-linked immunosorbent assay (ELISA) kits (Invitrogen, Life Technologies, Cergy Pontoise, France). Liver protein abundance of TNF$\alpha$ and IL-6 was also determined. See the Western blot (WB) procedure section. In addition, the gene expression of four major inflammatory actors (TNF- $\alpha$, IL-6, MCP-1, and CCL5) was also determined in the retroperitoneal adipose tissue. See the quantitative polymerase chain reaction (qPCR) procedure section for details.

\section{Hepatic Mitochondrial and NADPH Oxidase ROS Production}

Liver mitochondria were extemporaneously isolated using the differential centrifugation technique (Aoun et al., 2011). The final mitochondrial pellet was suspended in $1 \mathrm{~mL}$ mitochondrial respiration O5 (MIRO5) buffer (Sucrose $110 \mathrm{mM}$, Hepes $20 \mathrm{mM}, \mathrm{KH}_{2} \mathrm{PO}_{4} 10 \mathrm{mM}$, Taurine $20 \mathrm{mM}$, K-Lactobionate $60 \mathrm{mM}, \mathrm{MgCl}_{2} 3 \mathrm{mM}$ ethylene glycol-bis(2-aminoethylether)-N N $\quad \mathrm{N}^{\prime} \mathrm{N}^{\prime}$-tetraacetic acid (EGTA) $0.5 \mathrm{mM}$, bovine serum albumin (BSA) $1 \mathrm{~g} / \mathrm{L}$, $\mathrm{pH}$ 7.1). Protein content was determined using bovine serum albumin as standard (Bradford, 1976).
Mitochondrial ROS production was measured on fresh mitochondria as previously described (Barja, 2002). Briefly, reaction mixtures $(2 \mathrm{~mL})$, containing MIRO5 buffer, dihydrodichlorofluorescein $(10 \mu \mathrm{M})$, and peroxidase $(2 \mathrm{IU} / \mathrm{mL})$, were incubated with $50 \mu \mathrm{g}$ of mitochondrial proteins with or without substrates (malate/glutamate/succinate (S) $2.5 / 5 / 5 \mathrm{mM}$, respectively) and with or without adenine diphosphate (ADP) $(0.5 \mathrm{mM})$. Fluorescence was measured $60 \mathrm{~min}$ after reaction initiation at $\lambda_{\mathrm{Ex}}=490$ and $\lambda_{\mathrm{Ex}}=525 \mathrm{~nm}$ excitation and emission, respectively.

NADPH oxidase dependent-superoxide anion production was determined on rat liver as previously described (Feillet-Coudray et al., 2009). Briefly, liver homogenates were incubated at $37{ }^{\circ} \mathrm{C}$ for $30 \mathrm{~min}$ in the presence or absence of diphenyleneiodonium (DPI), a NADPH oxidase inhibitor. Lucigenin-enhanced chemiluminescence was assessed to determine $\mathrm{O}_{2}^{-}$generation after adding NADPH, the substrate for NADPH oxidase. The chemiluminescence signals were measured using a microplate luminometer LB96V (Berthold) for $30 \mathrm{~min}$. The results were expressed as the relative luminescence unit (RLU) and were corrected for protein concentration. In addition, the hepatic protein expression of two major NADPH oxidase subunits, p22 $2^{\text {phox }}$ and $\mathrm{p} 47^{\mathrm{phox}}$, was also determined. See the WB procedure section for details.

\section{Plasma and Liver Oxidative Stress Parameters}

Isoprostanoid metabolites measurement: To measure oxidative damage to lipids, the levels of isoprostanoids in plasma and liver were measured based on the micro-LC-MS/MS technique (Dupuy et al., 2016). Briefly, after lipid extraction with the Folch mixture, the extracts were mixed with a cocktail of internal standards, and an alkaline hydrolysis was performed. The metabolites were concentrated thanks to a solid phase extraction step conduced on weak-anion exchange materials. The metabolites were then analyzed using micro-LC-MS/MS. Mass spectrometry analysis was performed in an AB Sciex QTRAP5500 (Sciex Applied Biosystems). The ionization source was electrospray in negative mode. Detection of the fragmentation ion products from each deprotonated molecule was performed in the multiple reaction monitoring mode. Quantification of metabolites was done, using MultiQuant 3.0 software, by measuring the ratio of area under the specific metabolite peak/area under the internal standard peak and compared to the ratio of area under the metabolite calibration peak/area under the internal standard peak.

Liver protein carbonylation: The Oxyblot-Oxidized Protein Detection Kit was purchased from Chemicon (Hampshire, UK). The carbonyl groups in the protein side chains are derivatized to 2,4-dinitrophenol (DNP)hydrazone by reaction with 2,4-dinitrophenylhydrazine, 
following the manufacturer's instructions (Bravard et al., 2011). Briefly, after the derivatization of the protein sample, one-dimensional electrophoresis was carried out on a $10 \%$ sodium dodecyl sulfate polyacrylamide (SDS-PAGE) gel. Proteins were transferred to polyvinylidene difluoride membranes. After incubation with the anti-DNP antibody, the blot was developed using a chemiluminescence detection system. After transfers, the gels were stained with Coomassie Brilliant Blue to verify protein loading. These gels were scanned, and the intensity of each line allowed the normalization of the densitometry analysis of carbonylated proteins.

Long-established oxidative stress parameters: in addition to the isoprostanoid metabolites and protein carbonylation measurements, several long-established parameters of oxidative stress have also been evaluated. Thiobarbituric acid-reactive substances (TBARS), representing lipid peroxidation, were measured in plasma and liver homogenates by mixing the biological samples with thiobarbituric acid at $100{ }^{\circ} \mathrm{C}$ and monitoring the pink color absorbance at 532 nm (Sunderman et al., 1985; Yagi, 1984). Protein oxidation was assessed in plasma and liver supernatants by measuring the sulfhydryl groups using the Ellman reagent and monitoring the yellow color absorbance at $412 \mathrm{~nm}$ (Jocelyn, 1987). Plasma levels of advanced glycation end products (AGE) were evaluated monitoring the optical density at $430 \mathrm{~nm}$ after simple dilution in PBS-acetic acid buffer (Witko-Sarsat et al., 1998).

Total glutathione (GSH + GssG) and oxidized GSH were measured in blood and liver homogenates deproteinized with metaphosphoric acid. The blood and liver supernatants were mixed with the Ellman reagent and absorbance was monitored at $412 \mathrm{~nm}$ (Griffith, 1980). Total superoxide dismutase (SOD) was measured in plasma and liver supernatants by monitoring the inhibition of the auto-oxidation of pyrogall at $320 \mathrm{~nm}$ (Marklund, 1976). Catalase activity was measured in liver supernatants using $\mathrm{H}_{2} \mathrm{O}_{2}$ as a substrate and absorbance was monitored at $240 \mathrm{~nm}$ (Beers and Sizer, 1952). Glutathione peroxidase (GPx) was measured in plasma and liver supernatants using ter-butylhydroperoxide, GSH, GRx, and NADPH as substrates and monitoring the oxidation of NADPH at $340 \mathrm{~nm}$ (Flohe and Gunzler, 1984).

\section{Liver Protein Isolation and Western Blotting}

The hepatic abundance of major proteins involving inflammation and NADPH oxidase regulation was determined. Liver samples were homogenized using an Ultra Turax homogenizer in an ice cold lysis buffer: $20 \mathrm{mM}$ Tris $\mathrm{pH} 8$, $50 \mathrm{mM}$ DL-Dithiothreitol, $150 \mathrm{mM} \mathrm{NaCl}, 2 \mathrm{mM}$ EDTA, $1 \%$ Triton $\mathrm{X} 100,0.1 \%$ sodium dodecyl sulfate (SDS), $1 \mathrm{mM}$ phenylmethane sulfonyl fluoride (PMSF), $1 \mathrm{mM}$ orthovanadate, and $1 \%$ of antiprotease cocktail. Proteins were separated with $10 \%$ or $8 \%$ SDS-PAGE and then transferred to a nitrocellulose membrane. Membranes were blocked in $5 \%$ nonfat milk for $1 \mathrm{~h}$ at room temperature. Membranes were incubated overnight at $4{ }^{\circ} \mathrm{C}$ with a primary antibody against TNF- $\alpha$ (Abcam ab66579), IL-6 (Abcam ab25107), and subunits of NADPH oxidase $\mathrm{p} 22^{\text {phox }}$ (Abcam ab75941) and $\mathrm{p} 47^{\text {phox }}$ (Sigma-Aldrich SAB4502810) in blocking buffer. After washes in TBS/Tween buffer, membranes were incubated for $1 \mathrm{~h}$ with the horseradish peroxidase-labeled antibody. The antirabbit IgG, HRP-linked Antibody (Cell Signaling 7074) was used as a secondary antibody. After further washes, blots were treated with enhanced chemiluminescence detection reagents. $\beta$-actin (Santa Cruz sc-81,178) was used as loading references, and blot intensities were measured using the Image Labs software 5.2.1 (Bio-Rad Laboratories. France).

\section{Adipose Tissue RT-qPCR Analysis}

Real-time quantitative polymerase chain reaction (RTqPCR) was applied to measure key inflammation gens mRNA expression in the adipose tissue. Adipose tissue RNA was extracted with the Trizol reagent (Invitrogen Life Technologies, Cergy Pontoise, France). The reverse transcription reaction was performed with $1 \mu \mathrm{g}$ total RNA. Complementary deoxyribonucleic acid (cDNA) was synthesized with the use of moloney murine leukemia virus reverse transcriptase for first strand cDNA synthesis and random primers. The mRNA expressions of target genes (TNF- $\alpha$, IL-6, MCP-1, and CCL5) were determined by RTqPCR. RT-qPCR analysis was performed using SYBR Green Mastermix (Eurogentec, liege, Belgium) with a Mx3005P Real-Time PCR System (Stratagene, La Jolla, CA, USA). Results were normalized with the gene encoding $18 \mathrm{~S}$. The primer sequences used for real-time RT-PCR are given in Table 3 . The mean expression levels and the standard deviations of the housekeeping 18S rRNA gene were as follows: $5.56 \pm 0.17 ; 5.81 \pm 0.2 ; 5.78 \pm 0.25$; $5.84 \pm 0.42$; and $5.75 \pm 0.06$ for control groups $(4,8$, 12, 16, and 20 weeks, respectively) and $5.44 \pm 0.18$; $5.55 \pm 0.13 ; 5.49 \pm 0.15 ; 5.69 \pm 0.5$, and $5.53 \pm 0.190$ for HFHFr groups (4, 8, 12, 16, and 20 weeks, respectively).

\section{Statistical Analysis}

Results were expressed as means $\pm \mathrm{SD}, n=7-8$ animals per group per treatment duration. All the groups were tested for the effects of diet type, treatment duration, and their interaction by the two-way ANOVA test. When the effects of diet or treatment duration were significant, the unpaired Student's $t$-test was used to analyze the effect of HFHFr diet within each diet treatment duration point and the one- 
Table 3 Primer sequences for mRNA assays of inflammation genes for adipose tissue qPCR analyses

\begin{tabular}{lll}
\hline Gene & \multicolumn{1}{c}{ Forward Sequence $\left(5^{\prime}-3^{\prime}\right)$} & Reverse Sequence $\left(5^{\prime}-3^{\prime}\right)$ \\
\hline Tnfa & GCCCAACC CTCACACTC & CCACTCCAGCTGCTCCTCT \\
$I l 6$ & CCCTTCAGGAAC AGCTATGAA & ACAACATCAGTCCCAAGA AGG \\
Mcpl & AGCATCCACGTGCTGTCTC & GATCATCTTGCCAGTGAATGAGT \\
House Keeping Gene & CTCACCGTCATCCTCGTT G & GAGTGGTGTCCGAGCCATA \\
$18 S R N A$ & GTGGGCCTGCGGCTTAAT & GCCAGAGTCTCGTTCGTTATC \\
\hline
\end{tabular}

Tnfa: Tumor necrosis factor-a; Il6: interleukine-6; Mcpl (CCL2): Monocyte chemotactic protein-1; Ccl5: Chemokine (C-C motif) ligand 5; and 18S RRNA: 18S Ribosomal ribonucleic acid.

way ANOVA test was applied to analyze the effect of treatment duration for each diet followed up by a Fisher's Least Significant Difference test. The oral glucose tolerance test (OGTT) and the mitochondrial ROS production assessment were performed extemporaneously on $4,8,12,16$, and 20 weeks, and the WB analysis was carried out time point by time point. Therefore, the statistical analysis of these later data was made only between HFHFr and control diets, inside of each treatment duration, by the unpaired Student's $t$-test. The limit of statistical significance was set at $p<0.05$. HFHFr diet versus control diet: ${ }^{*} p<0.05$, $* * p<0.005$, and $* * * p<0.0005$. Correlations were performed with the Spearman method. Statistical analyses were performed using the StatView program (SAS Institute, Cary, NC, USA).

\section{Results}

\section{Hepatic Lipid Accumulation and Histological Analysis}

DAG and ceramide species are known as lipotoxic compounds and may have impact on hepatic insulin resistance. The liver content of total DAG was increased significantly $(+100 \%)$ under the HFHFr diet with a maximum on 16 weeks of diet intake compared to the control diet (Fig. 1a). Indeed, four DAG species were increased $(16 / 18,16 / 18: 1,18 / 18: 1$, and 18:1/18:1), whereas only one remained unchanged (16/16) and two were decreased (16/18:2 and 18:1/18:2) (Table S1, Supporting information).

In addition, the liver content of total ceramides was moderately but significantly increased under the HFHFr diet compared to the control diet (Fig. 1b). Indeed, five out of 10 ceramide species remained unchanged (SPA, Sph, 18:1, 24, and 24:1), whereas two were decreased (14 and 16) and three were increased $(18,20$, and 22). Therefore, the overall increase in the liver total ceramide content is due largely to the high increase in one of the major ceramide species, namely, the 22 species (Table S2).

As expected, the liver histological analysis, using hematoxylin/eosin staining, showed more lipid vesicles in the HFHFr diet-fed rats than in the control rats (Fig. 2a). The high score of these lipid vesicles in the HFHFr dietfed rats confirmed hepatic steatosis in this rat model (Fig. 2b). In addition, the plasma alanine aminotransferase (ALAT) activity, a marker of liver damage indicative of both cellular leakage and loss of liver cell membrane functional integrity, was significantly increased with the HFHFr diet compared to control diet (Fig. 2c).

\section{Blood and Tissue Inflammation Status}

The major cytokines TNF- $\alpha$, IL- 6 and the chemokines MCP-1 and CCL5, involved in systemic inflammation, were investigated in blood, liver, and/or adipose tissue. The plasma levels of both TNF- $\alpha$ and IL- 6 were increased throughout the study in both rat groups. However, the plasma levels of TNF- $\alpha$, IL-6, and MCP-1 remained unchanged between HFHFr and control groups (Table 4). In the liver tissue, the protein abundance of TNF- $\alpha$ remained unchanged whereas the protein abundance of IL6 was increased, with high variability (Table 4). In addition, the gene expression of two cytokines (Tnfa, Il6) and two chemokines ( $M c p 1, C c l 5)$ was also investigated in the adipose tissue. The expression of three genes out of four was increased significantly (Tnfa, Mcpl, and Ccl5) throughout the study in both rat groups. Nevertheless, only the gene expression of $\mathrm{Ccl} 5$ was highly increased with the HFHFr diets compared to the control diets (Table 4).

\section{Mitochondrial and NADPH Oxidase ROS Production}

Hepatic mitochondrial ROS production was evaluated on extemporaneously isolated liver mitochondria alone or challenged with substrates or with substrates plus ADP. As shown in Fig. 3a, mitochondrial ROS production remained substantially the same under the three experimental conditions. Interestingly, the NADPH oxidase dependentsuperoxide anion production was highly increased with the HFHFr diet compared to the control diet at the five explored time points (Fig. 3b). Nevertheless, the investigation of the two major proteins of hepatic NADPH oxidase, 
(a)

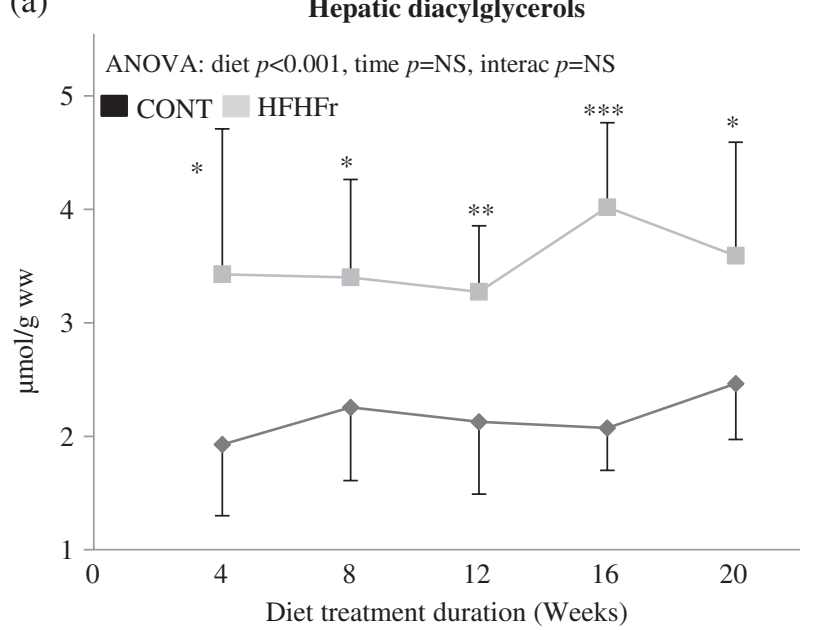

(b)

Hepatic ceramides

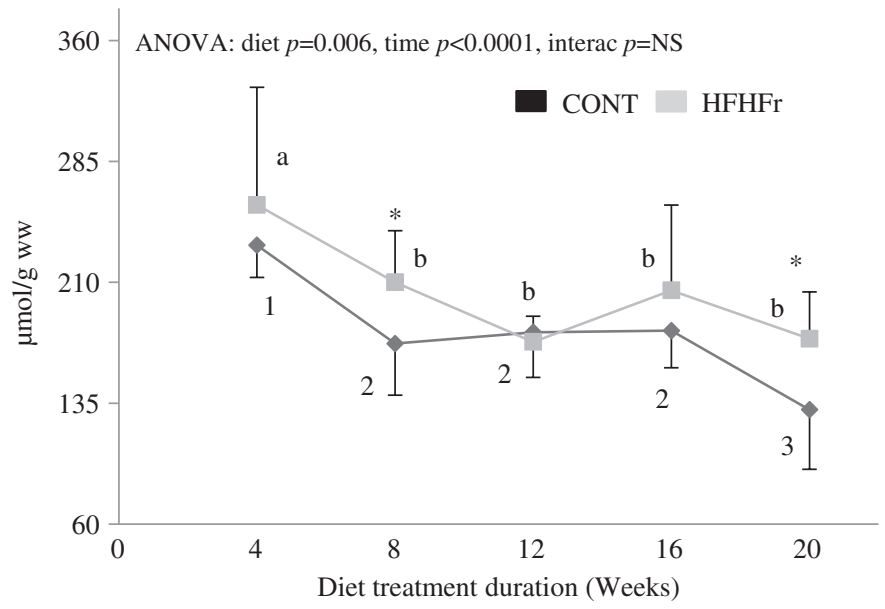

Fig. 1 Time-course of hepatic diacylglycerols (DAG) and ceramides accumulation. Results were expressed as means \pm SD, $n=6-8$ animals per group per diet treatment duration. Hepatic levels of putative mediators of lipid-induced hepatic insulin resistance diacylglycerols (a) and ceramides (b). All the groups were tested for the effects of diet, treatment duration, and their interaction using a two-way ANOVA. When the effects of diet or treatment duration were significant, the unpaired Student's $t$-test was used to analyze the effect of HFHFr diet within each diet treatment duration point and a one-way ANOVA was applied to analyze the effect of treatment duration for each diet followed up by a Fisher's Least Significant Difference test. The limit of statistical significance was set at $p<0.05$. High-fat high-fructose (HFHFr) diet versus control diet: $* p<0.05$, $* * p<0.005$, and $* * * p<0.0005$. Values with different superscript digits $(1,2,3$, and 4 for control groups) or with different superscript letters (a, $\mathrm{b}, \mathrm{c}, \mathrm{d}$, and e for HFHFr groups) are significantly different from each other $(p<0.05)$
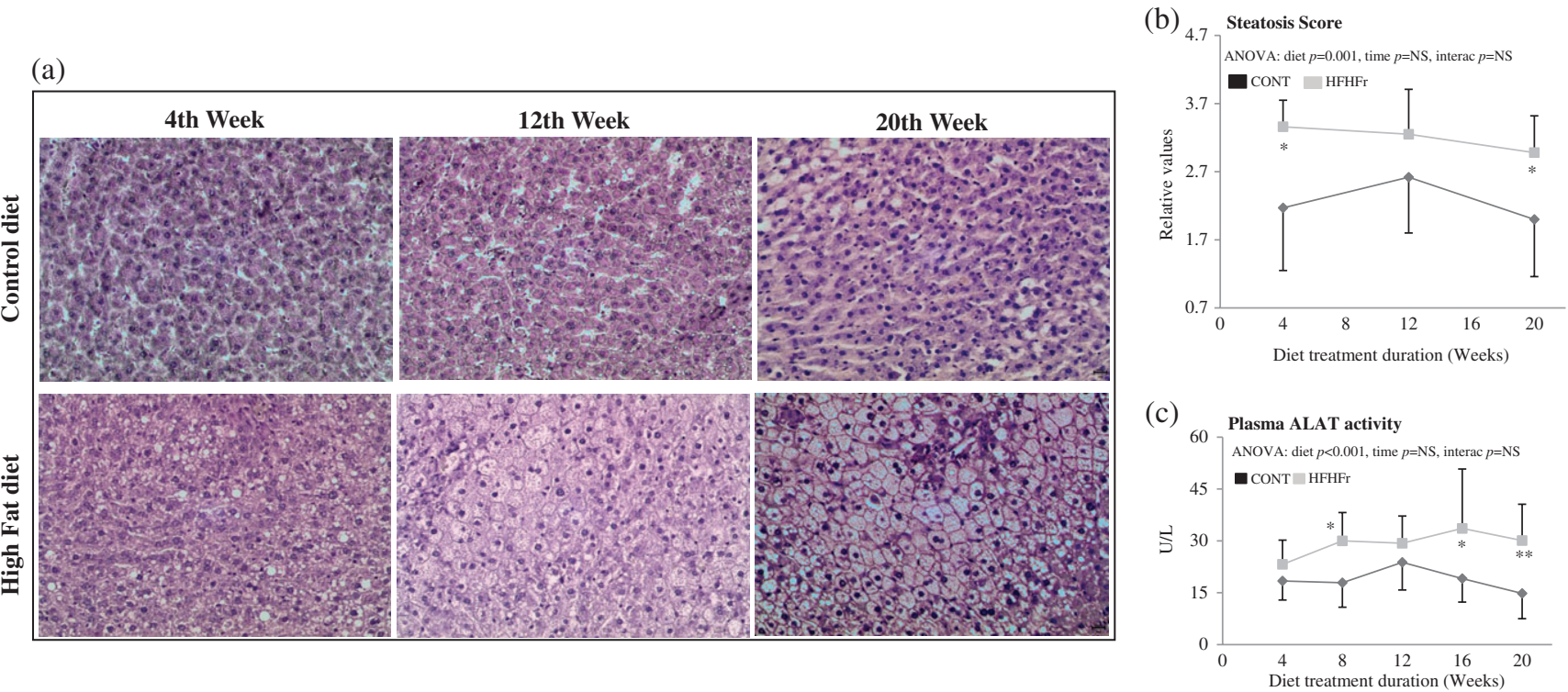

Fig. 2 Time-course of hepatic steatosis and plasma enzymatic ALAT activity. Results were expressed as means \pm SD, $n=6-8$ animals per group per diet treatment duration. Liver histology sections from a representative rat of both groups on the 4th, 12th, and the 20th weeks of diet, magnification 200x: (a). Histograms of the liver steatosis score showing higher hepatic lipid accumulation in the high-fat high-fructose (HFHFr) diet-fed rat compared to the control diet-fed rats (b). Plasma enzymatic ALAT activity (c). All the groups were tested for the effects of diet, treatment duration, and their interaction by two-way ANOVA. When the effects of diet or treatment duration were significant, the unpaired Student's $t$-test was used to analyze the effect of HFHFr diet within each diet treatment duration point and a one-way ANOVA was applied to analyze the effect of treatment duration for each diet followed up by a Fisher's least significant difference test. The limit of statistical significance was set at $p<0.05$. HFHFr diet versus control diet: ${ }^{*} p<0.05$, ${ }^{* *} p<0.005$. Values with different superscript digits $(1,2,3,4$ for control groups) or with different superscript letters (a, b, c, d, and e for HFHFr groups) are significantly different from each other $(p<0.05)$ 
Table 4 Blood, liver, and adipose tissue inflammatory status

\begin{tabular}{|c|c|c|c|c|c|c|c|c|c|}
\hline Parameter & Diet & 4 weeks & 8 weeks & 12 weeks & 16 weeks & 20 weeks & $p$ diet & $p$ time & $p$ interaction \\
\hline \multicolumn{10}{|l|}{ Plasma } \\
\hline \multirow[t]{2}{*}{ TNF- $\alpha(\mu \mathrm{g} / \mathrm{L})$} & Control & $5.8 \pm 0.6^{1}$ & $7.7 \pm 2.3^{2}$ & $11.4 \pm 1.1^{3}$ & $13.3 \pm 1.3^{4}$ & $14.5 \pm 0.9^{4}$ & & & \\
\hline & HFHFr & $6.5 \pm 1.1^{\mathrm{a}}$ & $8.1 \pm 1.4^{\mathrm{b}}$ & $11.2 \pm 0.6^{\mathrm{c}}$ & $13.2 \pm 1.7^{\mathrm{d}}$ & $14.6 \pm 1.1^{\mathrm{e}}$ & NS & $<0.0001$ & NS \\
\hline \multirow[t]{2}{*}{$\mathrm{IL}-6(\mu \mathrm{g} / \mathrm{L})$} & Control & $11.9 \pm 5.9^{1}$ & $19.1 \pm 7.7^{1,2}$ & $24.7 \pm 1.9^{2}$ & $34.6 \pm 8.6^{3}$ & $45.6 \pm 8.8^{4}$ & & & \\
\hline & HFHFr & $14.6 \pm 5.6^{\mathrm{a}}$ & $17.7 \pm 4.6^{\mathrm{a}}$ & $24.6 \pm 2.2^{\mathrm{b}}$ & $38.5 \pm 6.2^{\mathrm{c}}$ & $48.5 \pm 9.1^{\mathrm{d}}$ & NS & $<0.0001$ & NS \\
\hline \multirow[t]{2}{*}{ MCP-1 (ng/L) } & Control & $93 \pm 23$ & $112 \pm 34$ & $86 \pm 17$ & $118 \pm 33$ & $110 \pm 16$ & & & \\
\hline & HFHFr & $103 \pm 19$ & $90 \pm 13$ & $96 \pm 15$ & $106 \pm 17$ & $107 \pm 16$ & NS & NS & NS \\
\hline \multicolumn{10}{|l|}{ Liver } \\
\hline \multirow[t]{2}{*}{ TNF- $\alpha$, WB } & Control & $100 \pm 31$ & $100 \pm 47$ & $100 \pm 36$ & $100 \pm 035$ & $100 \pm 31$ & & & \\
\hline & HFHFr & $98 \pm 30$ & $93 \pm 15$ & $97 \pm 42$ & $86 \pm 27$ & $86 \pm 19$ & NA & NA & NA \\
\hline \multirow[t]{2}{*}{ IL-6, WB } & Control & $100 \pm 68$ & $100 \pm 60$ & $100 \pm 57$ & $100 \pm 47$ & $100 \pm 94$ & & & \\
\hline & HFHFr & $287 \pm 167^{*}$ & $351 \pm 254^{*}$ & $408 \pm 286^{*}$ & $265 \pm 118 * *$ & $320 \pm 215^{*}$ & NA & NA & NA \\
\hline \multicolumn{10}{|l|}{ Adipose tissue } \\
\hline \multirow[t]{2}{*}{ Tnf- $\alpha$, qPCR } & Control & $100 \pm 120^{1,2}$ & $77 \pm 36^{1}$ & $59 \pm 39^{1}$ & $115 \pm 54^{1,2}$ & $146 \pm 36^{2}$ & & & \\
\hline & HFHFr & $84 \pm 34^{\mathrm{a}}$ & $76 \pm 12^{\mathrm{a}}$ & $79 \pm 28^{\mathrm{a}}$ & $129 \pm 54^{\mathrm{b}}$ & $152 \pm 64^{\mathrm{b}}$ & NS & 0.0011 & NS \\
\hline \multirow[t]{2}{*}{ Il-6, qPCR } & Control & $100 \pm 92$ & $62 \pm 62$ & $188 \pm 32$ & $149 \pm 84$ & $212 \pm 101$ & & & \\
\hline & HFHFr & $37 \pm 34$ & $80 \pm 83$ & $117 \pm 144$ & $136 \pm 108$ & $194 \pm 296$ & NS & 0.0827 & NS \\
\hline \multirow[t]{2}{*}{ Mcp-1, qPCR } & Control & $100 \pm 50^{1}$ & $102 \pm 61^{1}$ & $126 \pm 79^{1}$ & $168 \pm 101^{1,2}$ & $248 \pm 160^{2}$ & & & \\
\hline & HFHFr & $84 \pm 37^{\mathrm{a}}$ & $131 \pm 55^{\mathrm{a}}$ & $131 \pm 96^{\mathrm{ab}}$ & $164 \pm 81^{\mathrm{bc}}$ & $216 \pm 107^{\mathrm{c}}$ & NS & 0.0009 & NS \\
\hline \multirow[t]{2}{*}{ Ccl5, qPCR } & Control & $100 \pm 36^{1}$ & $73 \pm 69^{1}$ & $53 \pm 40^{1}$ & $101 \pm 66^{1}$ & $126 \pm 148^{1}$ & & & \\
\hline & HFHFr & $66 \pm 33^{a}$ & $146 \pm 115^{\mathrm{a}}$ & $198 \pm 111^{* \mathrm{a}}$ & $323 \pm 162 * * \mathrm{~b}$ & $440 \pm 273^{* \mathrm{~b}}$ & 0.0001 & 0.0038 & 0.0206 \\
\hline
\end{tabular}

Results were expressed as means $\pm \mathrm{SD}, n=6-8$ animals per group per treatment duration. All the groups were tested for the effects of diet, treatment duration, and their interaction using a two-way ANOVA. When the effects of diet or treatment duration were significant, the unpaired Student's $t$-test was used to analyze the effect of high-fat high-fructose (HFHFr) diet within each diet treatment duration point and the one-way ANOVA test was applied to analyze the effect of treatment duration for each diet followed up by a Fisher's Least Significant Difference test. The limit of statistical significance was set at $p<0.05$. HFHFr diet versus control diet: $* p<0.05,{ }^{*} p<0.005$. Values in the same row with different superscript digits $(1,2,3$, and 4 for control groups) or with different superscript letters (a, b, c, d, and e for HFHFr groups) are significantly different from each other $(p<0.05)$. NS: not significant; NA: non applicable. TNF- $\alpha$ : tumor necrosis factor- $\alpha$; Il-6: interleukine 6; Mcp-1: monocyte chemoattractant protein 1; and Ccl5: chemokine (C-C motif) ligand 5.

$\mathrm{p} 22^{\text {phox }}$ and $\mathrm{p} 47^{\text {phox }}$, did not indicate any significant difference between HFHFr and control groups at any treatment duration (Fig. 3b).

\section{Plasma and Liver Isoprostanoid Contents}

This study consists of LC-MS profiling of 14 isoprostanoid metabolites in plasma and in liver. We have classified the measured isoprostanoid metabolites into six classes according to their fatty acid origin: class A: radical metabolites of $\alpha$-linolenic acid (18:3 n-3), class B: radical metabolites of arachidonic acid (20:4 n-6), class C: radical metabolites of all-cis-7,10,13,16-docosatetraenoic acid (adrenic acid) (22:4 n-6), class D: radical metabolites of docosapentaenoic $\operatorname{acid}_{n-6}(22: 5 n-6)$, class E: radical metabolites of docosahexaenoic acid (22:6 n-3), and class F: enzymatic metabolites of arachidonic acid (20:4 n-6).

If the plasma isoprostanoid content of class $\mathrm{A}$ and $\mathrm{F}$ remained unchanged (Fig. 4a, e), the plasma isoprostanoid contents of classes B, C, and E were decreased significantly under the HFHFr diet compared to the control diet
(Fig. 4b-d). The metabolite of the class D (F3-neuroprostane) was not detectable. Consequently, the plasma total isoprostanoid content was decreased significantly under the HFHFr diet compared to the control diet (Fig. 4f). Such a decrease was marked from the 12 weeks of diet intake (Fig. 4f). The plasma individual isoprostanoid metabolites are shown in Table S3.

The liver isoprostanoid contents of the classes A, B, C, and $\mathrm{E}$ were decreased significantly under the HFHFr diet compared to the control diet (Fig. 5a, b, c, e). Only the F3 t-neuroprostane metabolite was increased (class D, Fig. 5d). Therefore, the liver total isoprostanoid content was decreased significantly under the HFHFr diet compared to the control diet. Such a decrease was observed on 12, 16, and 20 weeks of diet intake (Fig. 5f). The liver individual isoprostanoid metabolites are shown in Table S4.

As the hepatic isoprostanoid content may depend on the hepatic levels of their original fatty acids, we have also looked at the ratio of liver isoprostanoid/original fatty acid of the isoprostanoid classes as well as at the ratio of total isoprostanoid/ total PUFA. Interestingly, the ratio of isoprostanoid/fatty acid 

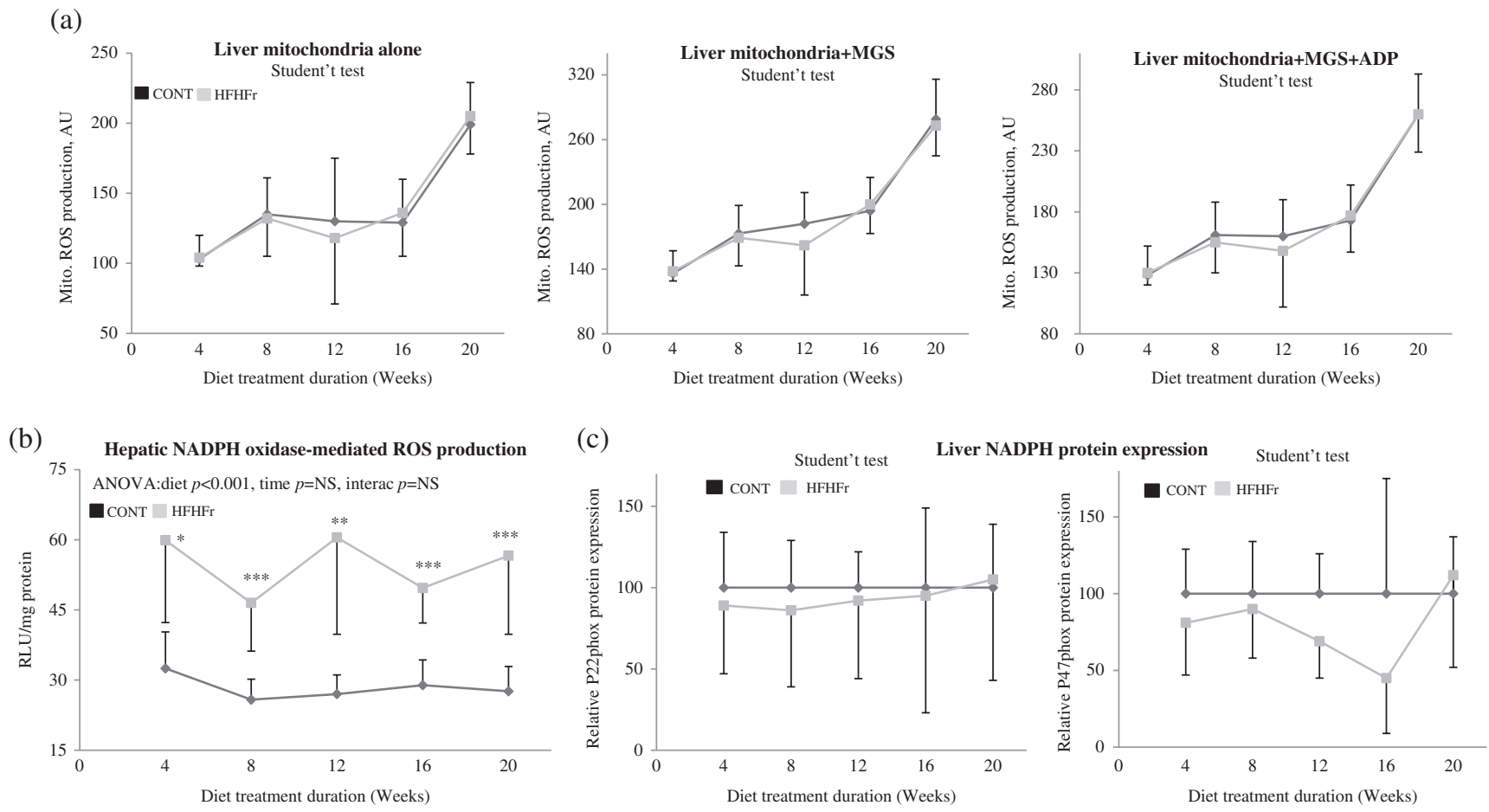

Fig. 3 Liver mitochondrial-mediated and NADPH oxidase-mediated reactive oxygen species (ROS) production and NADPH protein expression. Results were expressed as means $\pm \mathrm{SD}, n=6-8$ animals per group per diet treatment duration. Liver mitochondrial ROS production alone or challenged with substrates (M/G/S) or with substrates plus adenine diphosphate (ADP) (a), Liver NADPH oxidase-mediated ROS production (b), and hepatic protein abundance of $\mathrm{p} 22^{\mathrm{phox}}$ and $\mathrm{p} 47^{\text {phox }}(\mathrm{c})$. All the groups were tested for the effects of diet, treatment duration, and their interaction using a two-way ANOVA. When the effects of diet or treatment duration were significant, the unpaired Student's $t$-test was used to analyze the effect of high-fat high-fructose (HFHFr) diet within each diet treatment duration point and a one-way ANOVA was applied to analyze the effect of treatment duration for each diet followed up by a Fisher's least significant difference test. The limit of statistical significance was set at $p<0.05$. HFHFr diet versus control diet: $* p<0.05, * * p<0.005$, and $* * * p<0.0005$. Values with different superscript digits $(1,2,3,4$ for control groups) or with different superscript letters (a, b, c, d, and e for HFHFr groups) are significantly different from each other $(p<0.05)$. ROS, reactive oxygen species; MGS, malate+glutamate+succinate; RLU, relative luminescence unit

for the isoprostanoid classes $\mathrm{C}$ and $\mathrm{E}$ as well as for the ratio total isoprostanoids/total PUFA remained lower with the HFHFr diet than with the control diet (Table S5).

\section{Long-Established Oxidative Stress Parameters}

As for isoprostanoids, plasma TBARS levels were decreased moderately but significantly under the HFHFr diet compared to the control diet, in particular on 4, 12, and 16 weeks of diet (Fig. 6a). However, plasma AGE levels were significantly increased with the HFHFr diet compared to the control diet on 8 and 12 weeks of diet (Fig. 6b). Plasma - SH group levels were significantly decreased with the HFHFr diet compared to the control diet (Fig. 6c), whereas total GSH, GssG levels, and GssG/GSH ratio remained unchanged throughout the study between the two experimental diets (Fig. 6d). Finally, plasma antioxidant enzymes SOD and GPx (Fig. 6e, f) were significantly decreased throughout the study in both diet groups, and the SOD activity was moderately increased with the HFHFr diet compared to the control diet.
The hepatic oxidative stress was more marked than blood oxidative stress. The liver protein carbonylation measured with the Oxyblot kit remained unchanged throughout the study between the two experimental diets but was significantly increased with the HFHFr diet compared to the control diet (Fig. 7a, b). However, liver TBARS levels were increased throughout the study but remained statistically unchanged between the two experimental diets (Fig. 8a). Nevertheless, liver contents of $-\mathrm{SH}$ groups and total GSH and GssG were markedly decreased with the HFHFr diet compared to the control diet (Fig. 8b). Moreover, the catalytic activities of the antioxidant enzymes SOD and GPx, but not catalase, were decreased with the HFHFr diet compared to the control diet (Fig. 8c).

\section{Relationships between Glucose Intolerance and Oxidative Stress Parameters}

In attempt to understand the relationship between glucose intolerance and oxidative stress, correlations between the area under the curve (AUC) of the OGTT and oxidative 

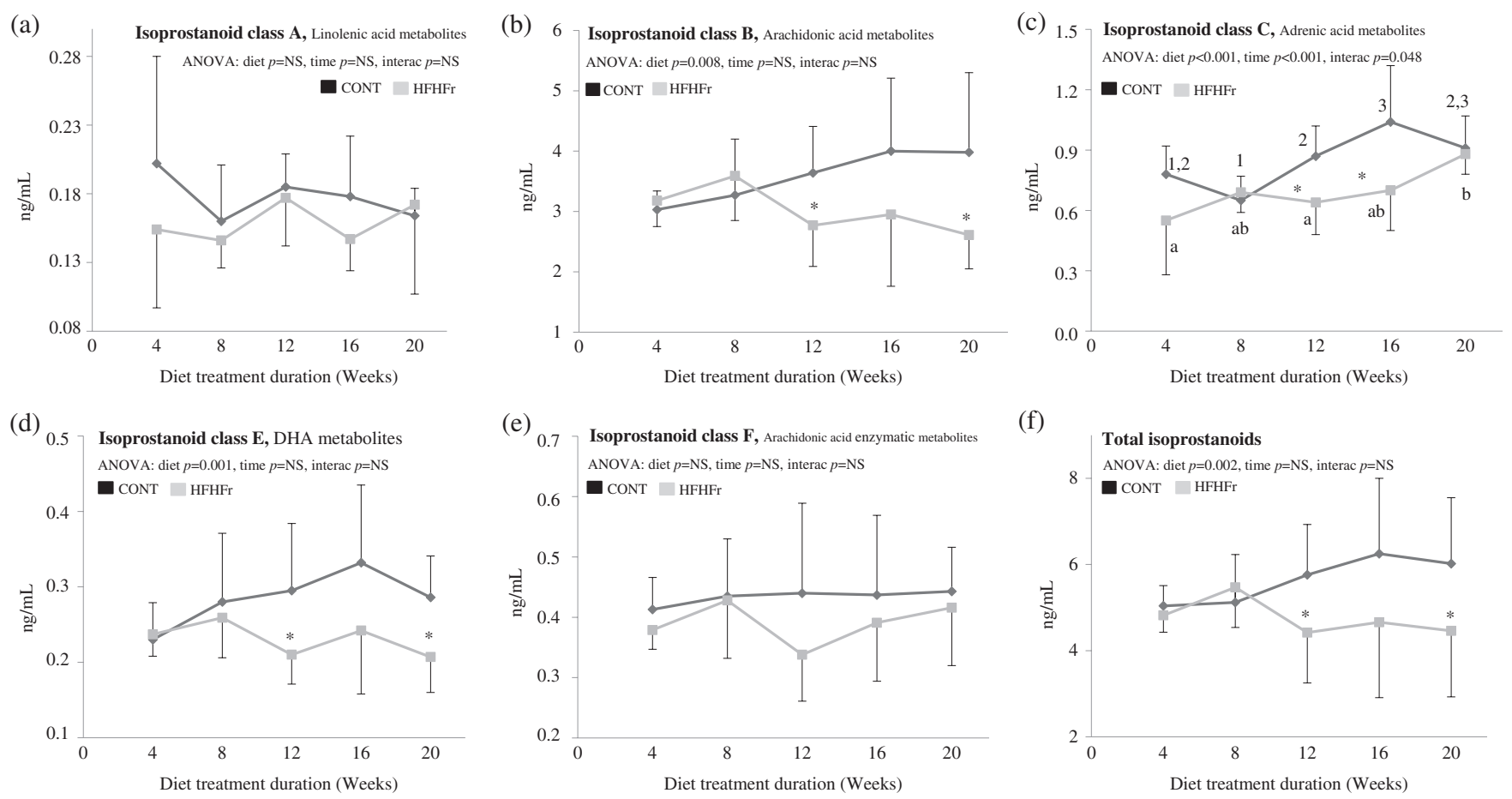

Fig. 4 Plasma isoprostanoid contents and their major classes. Results were expressed as means $\pm \mathrm{SD}, n=6-8$ animals per group per diet treatment duration. Plasma isoprostanoid class A (a), isoprostanoid class B (b), isoprostanoid class C (c), isoprostanoid class E (d), isoprostanoid class F (e), and total isoprostanoid levels (f). All the groups were tested for the effects of diet, treatment duration, and their interaction using a two-way ANOVA. When the effects of diet or treatment duration were significant, the unpaired Student's $t$-test was used to analyze the effect of high-fat high-fructose (HFHFr) diet within each diet treatment duration point and a one-way ANOVA was applied to analyze the effect of treatment duration for each diet followed up by a Fisher's least significant difference test. The limit of statistical significance was set at $p<0.05$. HFHFr diet versus control diet: $* p<0.05$. Values with different superscript digits $(1,2,3$, and 4 for control groups) or with different superscript letters (a, b, $\mathrm{c}, \mathrm{d}$, and e for HFHFr groups) are significantly different from each other $(p<0.05)$. Isoprostanoid classes: class A: radical metabolites of $\alpha$-linolenic acid (18:3 n-3), class B: radical metabolites of arachidonic acid (20:4 n-6), class C: radical metabolites of adrenic acid (22:4 n-6), class D: (nondetectable); class E: radical metabolites of docosahexaenoic acid (22:6 n-3), and class F: enzymatic metabolites of arachidonic acid (20:4 n-6)

stress parameters have been sought (Fig. 9). There was a negative correlation between the AUC and the plasma and hepatic levels of sulfhydryl groups $(r=-0.332$, $p=0.0042$ and $r=-0.391$, and $p=0.0007$, respectively). Surprisingly, there was also a negative correlation between AUC and plasma and hepatic contents of isoprostanoids $(r=-0.285, p=0.0155$ and $r=-0.368, p=0.0012$, respectively). There was also a moderate negative correlation between the AUC and the mitochondria+substrates $+\mathrm{ADP}(r=-0.265, p=0.0218)$ and a positive correlation with NADPH oxidase activity $(r=0.367, p=0.0015)$.

\section{Discussion}

The present work was designed to determine the coursetime evolution of both inflammation and oxidative stress and their relation to the major MetS features, namely, glucose intolerance and hepatic steatosis development, in an HFHFr diet-induced oxidative stress model in a large chronic study with several end points from 4-week to 20-week diet treatment. In a recent study (Fouret et al., 2018), we have reported that HFHFr diet, rich in fat and fructose that imitates western diet, has been efficient in inducing moderate overweight, increased liver and adipose tissue weights, and hyperglycemia from the $8 \mathrm{th} / 12 \mathrm{th}$ week of diet. The results of the present study are an extension of the previous article that investigated hepatic accumulation of lipotoxic metabolites as well as inflammation and oxidative stress in these rats fed a high-fat/highfructose diet.

Many studies have demonstrated increased accumulation of lipids in the liver in mice and rats receiving HF diet. However, it is the intracellular lipotoxic metabolites (DAG and ceramides) that are considered to be the true culprits of the initiation and the development of glucose intolerance and hepatic steatosis. Indeed, in addition to the marked increase in liver triacylglycerols (TAG) and cholesterol ester contents, our results have shown that the hepatic contents of DAG species were markedly increased with the HFHFr diet compared to the control diet and this was 

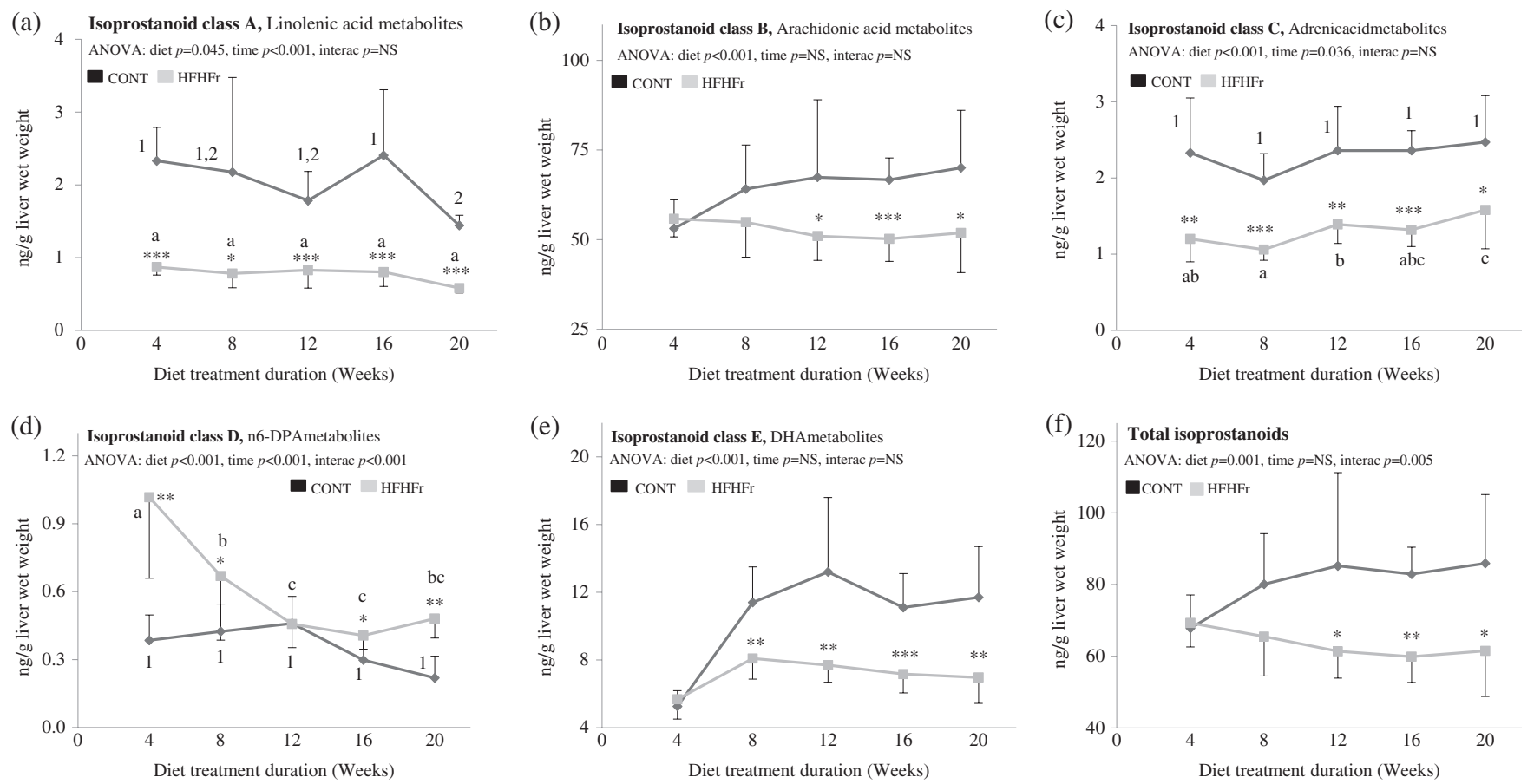

Fig. 5 Liver total isoprostanoid contents and their major classes, ng/g liver wet weight. Results were expressed as means \pm SD, $n=6-8$ animals per group per diet treatment duration. Hepatic isoprostanoid class A (a), isoprostanoid class B (b), isoprostanoid class C (c), isoprostanoid class D (d), isoprostanoid class E (e), and total isoprostanoid contents (f). All the groups were tested for the effects of diet, treatment duration, and their interaction using a two-way ANOVA. When the effects of diet or treatment duration were significant, the unpaired Student's $t$-test was used to analyze the effect of high-fat high-fructose (HFHFr) diet within each diet treatment duration point and a one-way ANOVA was applied to analyze the effect of treatment duration for each diet followed up by a Fisher's least significant difference test. The limit of statistical significance was set at $p<0.05$. HFHFr diet versus control diet: $* p<0.05, * * p<0.005$, and $* * * p<0.0005$. Values with different superscript digits $(1,2,3$, and 4 for control groups) or with different superscript letters (a, b, c, d, and e for HFHFr groups) are significantly different from each other $(p<0.05)$. Isoprostanoid classes: class A: radical metabolites of $\alpha$-linolenic acid $(18: 3 n-3)$, class B: radical metabolites of arachidonic acid $(20: 4$ $\mathrm{n}-6)$, class C: radical metabolites of adrenic acid (22:4 n-6), class D: radical metabolites of docosapentaenoic acid $\mathrm{n}-6$ (22:5 n-6), and class E: radical metabolites of docosahexaenoic acid (22:6 n-3)

observed at the five experimental time points of our present study. A variety of factors are responsible for the ectopic accumulation of DAG in the liver. These especially include the consumption of a diet rich in fats or sugars as well as the inability of adipose tissue to store lipids appropriately, resulting in increased circulating unesterified fatty acids. Indeed, hepatic DAG accumulation in both animals and humans has been linked to hepatic insulin resistance (Finck and Hall, 2015).

It is generally admitted that accumulation of ectopic fat in the liver and resulting hepatic insulin resistance results in hepatic inflammation, secondary to cytokine release either locally by the liver or by the white adipose tissue. (Peng et al., 2012). Some cytokines may exacerbate NAFLD and insulin resistance, such as TNF- $\alpha$ or IL-6 and others may compensate the situation, such as IL-10 and adiponectin (Asrih and Jornayvaz, 2013). In this study, many inflammatory cytokines we measured in blood, liver, and adipose tissue. Our results showed that hepatic protein abundance of IL- 6 and adipose tissue gene expression of chemokine $(\mathrm{C}-\mathrm{C}$ motif) ligand 5 ( $\mathrm{Ccl} 5)$ was significantly increased with the HFHFr diet compared to the control diet. However, the other inflammation markers (plasma TNF- $\alpha$ and IL-6, liver TNF- $\alpha$, adipose tissue Tnf$\alpha$, Il-6, and Mcp-1) remained unchanged. Therefore, only limited inflammation was observed in our HFHFr rats. Indeed, although low-grade inflammation may accompany steatosis, frank inflammation is usually observed in the later stages of steatosis, which, along with oxidative stress, is responsible for the progression of steatosis to steatohepatitis and fibrosis (Nakamura and Terauchi, 2013; Tilg and Moschen, 2010).

The critical roles of cellular ROS and oxidative stress involved in the pathogenesis of glucose intolerance and liver diseases have been highlighted for decades, and accumulating evidence showed that a vicious cycle could be created by oxidative stress and inflammation. Here, we investigated the two major sources of hepatic ROS production, namely, mitochondria and NADPH oxidase. Under our experimental conditions, we did not observe any difference in the hepatic mitochondrial ROS production among the studied groups; even though this does not quite exclude 

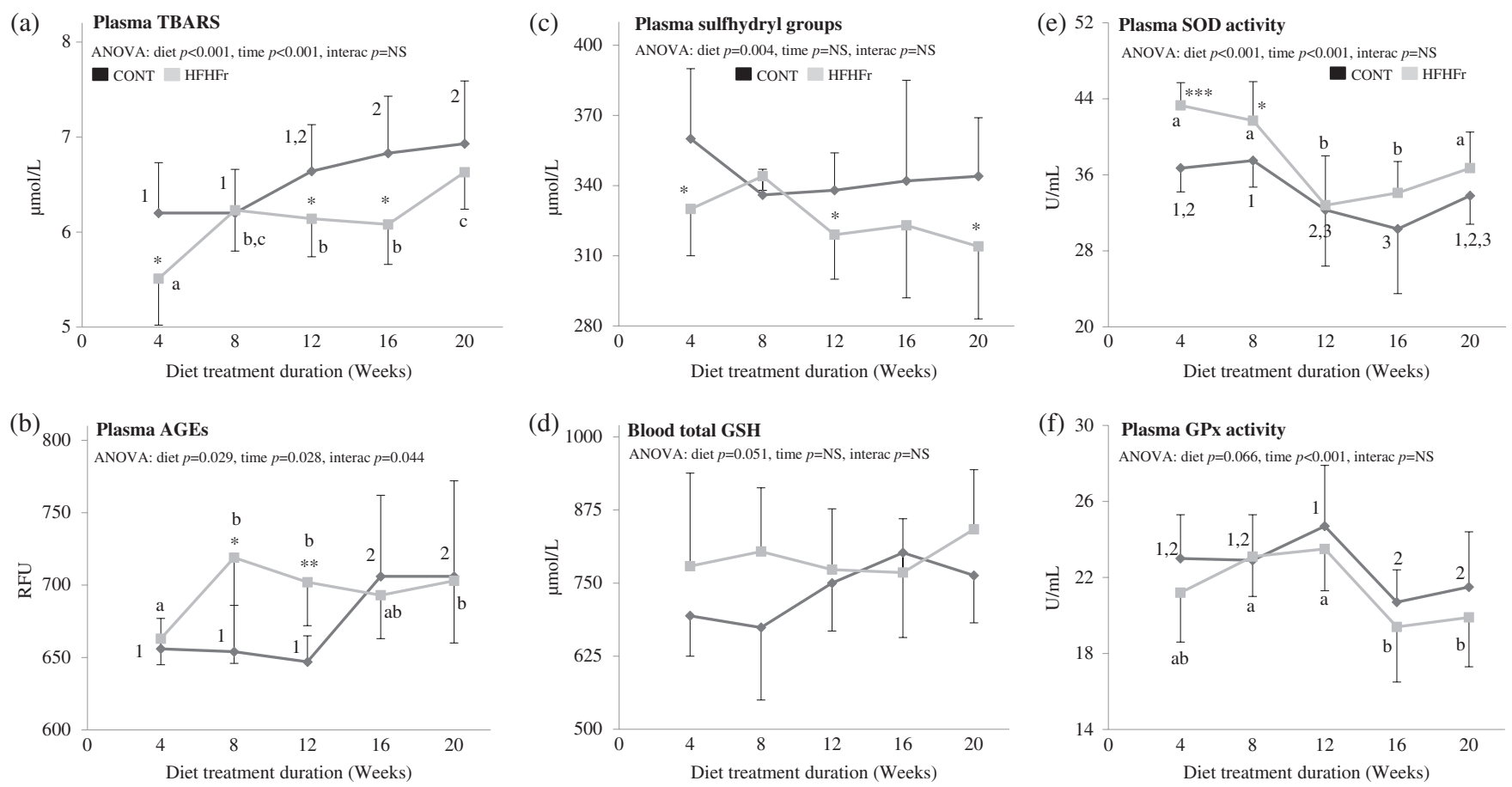

Fig. 6 Blood long-established oxidative stress markers. Results were expressed as means \pm SD, $n=6-8$ animals per group per diet treatment duration. Blood oxidative Plasma thiobarbituric acid-reactive substance [TBARS] (a) and advanced glycation end products [AGE] (b) and antioxidative stress (plasma - SH groups (c), blood glutathione [GSH] (d), and plasma superoxide dismutase [SOD] (e) and plasma glutathione peroxidase [GPx] (f)) parameters were determined. All the groups were tested for the effects of diet, treatment duration, and their interaction using a two-way ANOVA. When the effects of diet or treatment duration were significant, the unpaired Student's $t$-test was used to analyze the effect of high-fat high-fructose (HFHFr) diet within each diet treatment duration point and a one-way ANOVA was applied to analyze the effect of treatment duration for each diet followed up by a Fisher's least significant difference test. The limit of statistical significance was set at $p<0.05$. HFHFr diet versus control diet: ${ }^{*} p<0.05, * * p<0.005$. Values with different superscript digits $(1,2,3$, and 4 for control groups) or with different superscript letters ( $\mathrm{a}, \mathrm{b}, \mathrm{c}, \mathrm{d}$, and e for HFHFr groups) are significantly different from each other $(p<0.05)$. RFU: relative fluorescence unit; - SH groups: sulfhydryl groups

(a)

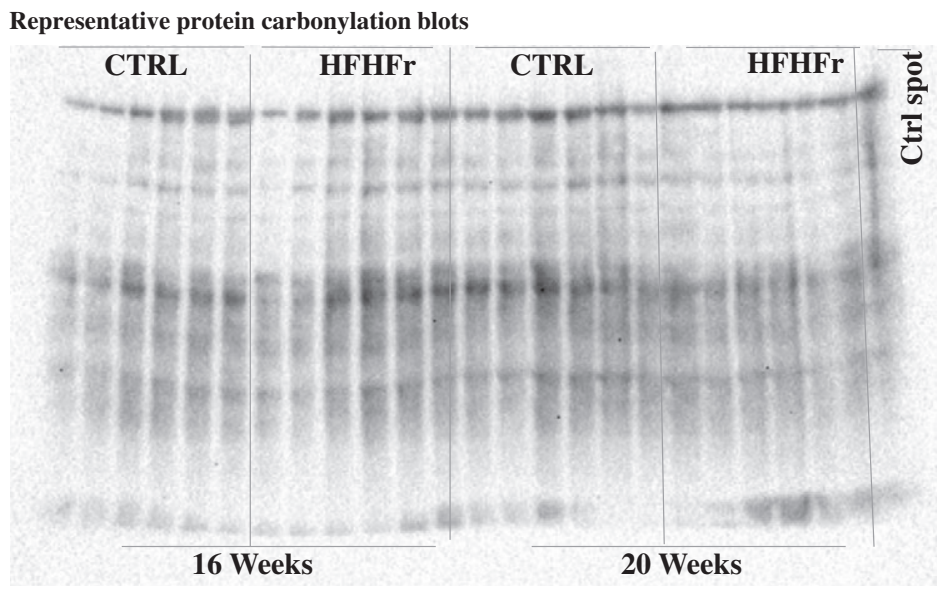

(b)

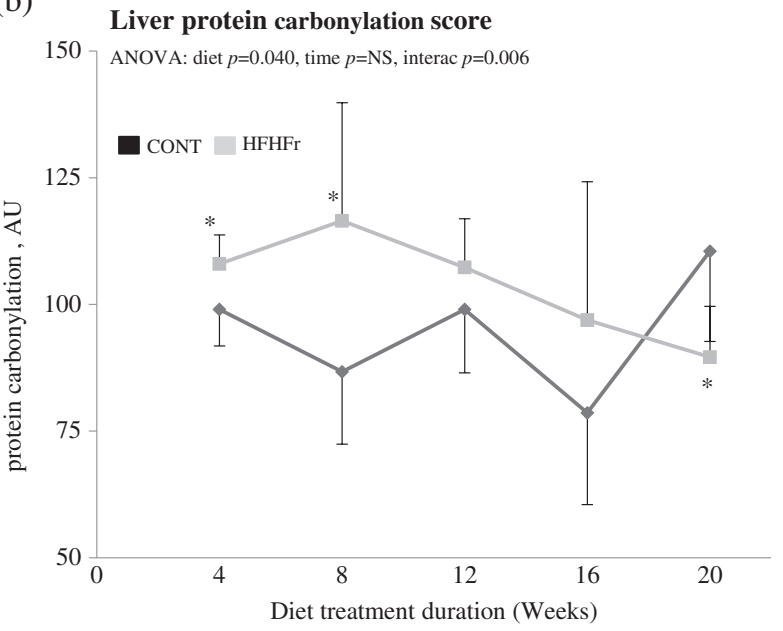

Fig. 7 Liver protein carbonylation. Results were expressed as means $\pm \mathrm{SD}, n=6-8$ animals per group per diet treatment duration. Representative liver protein carbonylation blots (a) and liver protein carbonylation score (b). All the groups were tested for the effects of diet, treatment duration, and their interaction using a two-way ANOVA. When the effects of diet or treatment duration were significant, the unpaired Student's $t$-test was used to analyze the effect of high-fat high-fructose (HFHFr) diet within each diet treatment duration point and a one-way ANOVA was applied to analyze the effect of treatment duration for each diet followed up by a Fisher's least significant difference test. The limit of statistical significance was set at $p<0.05$. HFHFr diet versus control diet (student $t$-test): ${ }^{*} p<0.05$. Values with different superscript digits $(1,2,3$, and 4 for control groups) or with different superscript letters ( $\mathrm{a}, \mathrm{b}, \mathrm{c}, \mathrm{d}$, and e for HFHFr groups) are significantly different from each other $(p<0.05)$ 
(a)

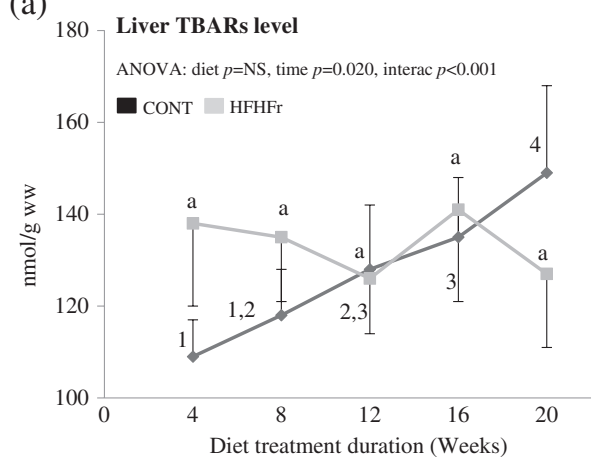

(c)

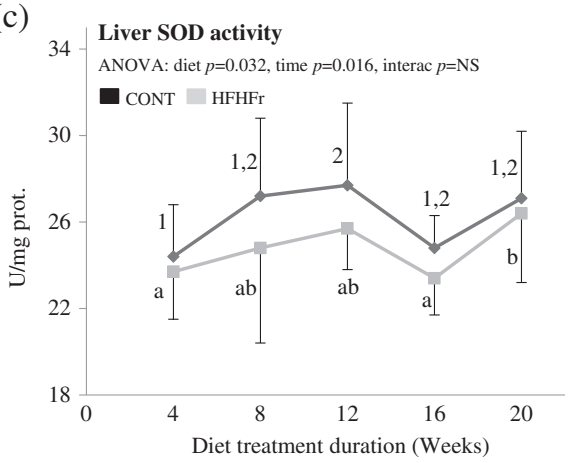

(b)
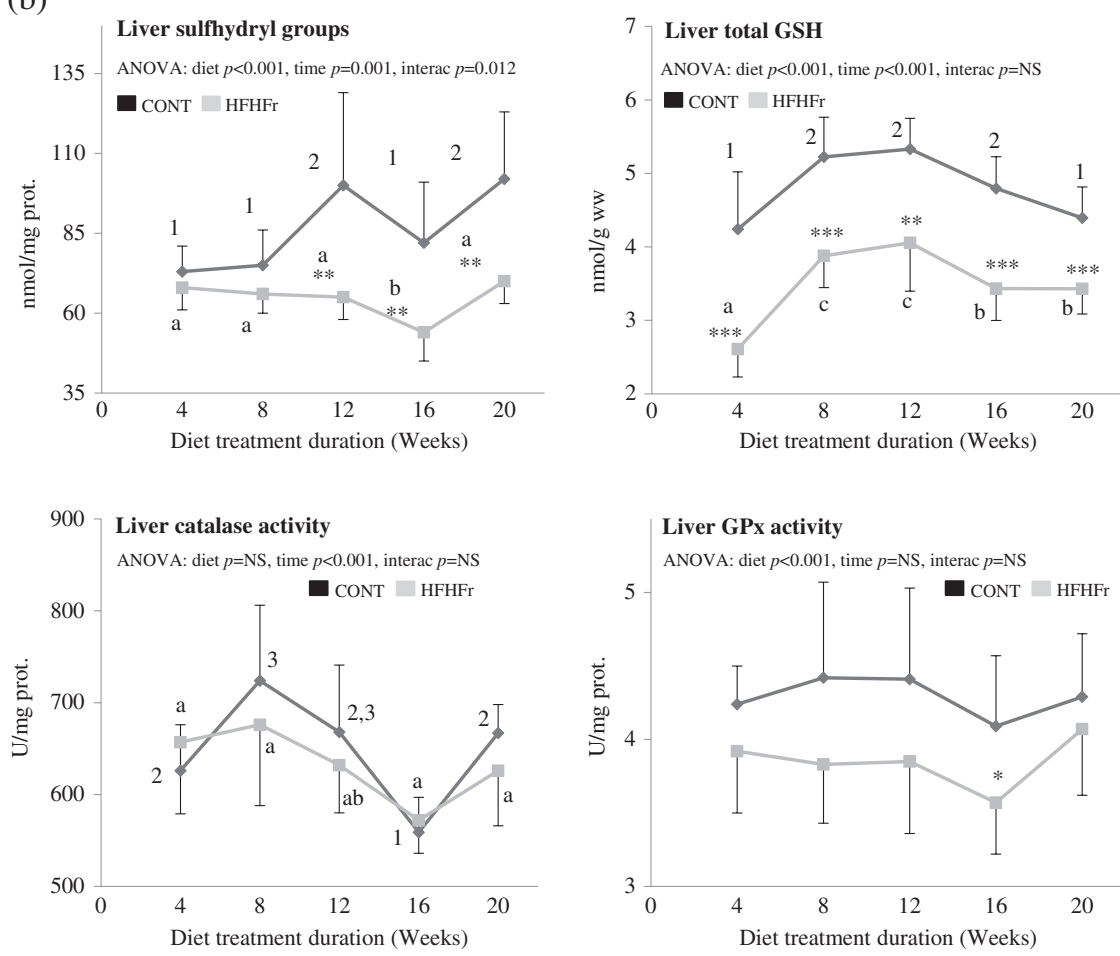

Fig. 8 Long-established oxidative stress markers of liver. Results were expressed as means $\pm \mathrm{SD}, n=6-8$ animals per group per diet treatment duration. Hepatic oxidative, thiobarbituric acid-reactive substance [TBARS] (a), antioxidative stress, $-\mathrm{SH}$ groups, total glutathione [GSH] (b), and superoxide dismutase [SOD], catalase and glutathione peroxidase [GPx] (c) parameters were determined. All the groups were tested for the effects of diet, treatment duration, and their interaction using a two-way ANOVA. When the effects of diet or treatment duration were significant, the unpaired Student's $t$-test was used to analyze the effect of high-fat high-fructose (HFHFr) diet within each diet treatment duration point and a one-way ANOVA was applied to analyze the effect of treatment duration for each diet followed up by a Fisher's least significant difference test. The limit of statistical significance was set at $p<0.05$. HFHFr diet versus control diet: $* p<0.05, * * p<0.005$, and $* * * p<0.0005$. Values with different superscript digits (1,2,3,4 for control groups) or with different superscript letters (a, b, c, d, and e for HFHFr groups) are significantly different from each other $(p<0.05)$. - SH groups: sulfhydryl groups

the possibility that in vivo mitochondrial ROS production may be affected. Indeed, in vitro measurement of ROS in isolated mitochondria can skew it because it deprives mitochondria of their natural environment within the cell. Higher mitochondrial ROS production with HF diets was shown only when using palmitoyl-carnitine as substrates, but not when using malate/glutamate/succinate as substrates as in our study (Vial et al., 2011). However, another team (Cardoso et al., 2010) has found that a high-fat diet increased $\mathrm{H}_{2} \mathrm{O}_{2}$ release from mice liver mitochondria without substantial alteration in respiratory rates, ADP/O ratios, or membrane potentials of isolated liver mitochondria. It is highly likely that there are different responses to high-fat diets between rats and mice.

The NADPH oxidase system is another important source of cellular ROS production and an essential component of redox signaling mechanisms, and so it seems to play a major role in the pathogenesis of oxidative metabolic disorders (Furukawa et al., 2004; Roberts et al., 2006). Indeed, an abnormal activation of NADPH oxidase is accompanied by high production of superoxide anions, which is generally related to tissue injury induced by oxidative stress. In the present study, liver NADPH oxidase dependent-superoxide anion production was markedly increased with the HFHFr diet compared to the control diet and this was at the four measured time points. However, the protein abundance of two major NADPH oxidase components, $\mathrm{p} 22^{\text {phox }}$ and $\mathrm{p} 47^{\text {phox }}$, remained statistically unchanged with the HFHFr diet compared to the control diet. Indeed, the observed higher NADPH oxidase activity with the HFHFr diet may result from post-translational modifications (phosphorylation, etc.) followed by membrane $\mathrm{p} 47^{\text {phox }}$ translocation and NADPH oxidase activation (Brandes et al., 2014). As shown in Fig. 9f, NADPH oxidase activity correlated highly positively with the glucose intolerance test. These results suggested that NADPH oxidase-mediated oxidative stress may contribute to glucose intolerance and liver injury during high-fat diet feeding. In addition and in agreement with such hypothesis, a novel NADPH oxidase inhibitor, the GLX351322, was reported to counteract glucose intolerance in high-fat diettreated C57BL/6 mice (Anvari et al., 2015). Another team 

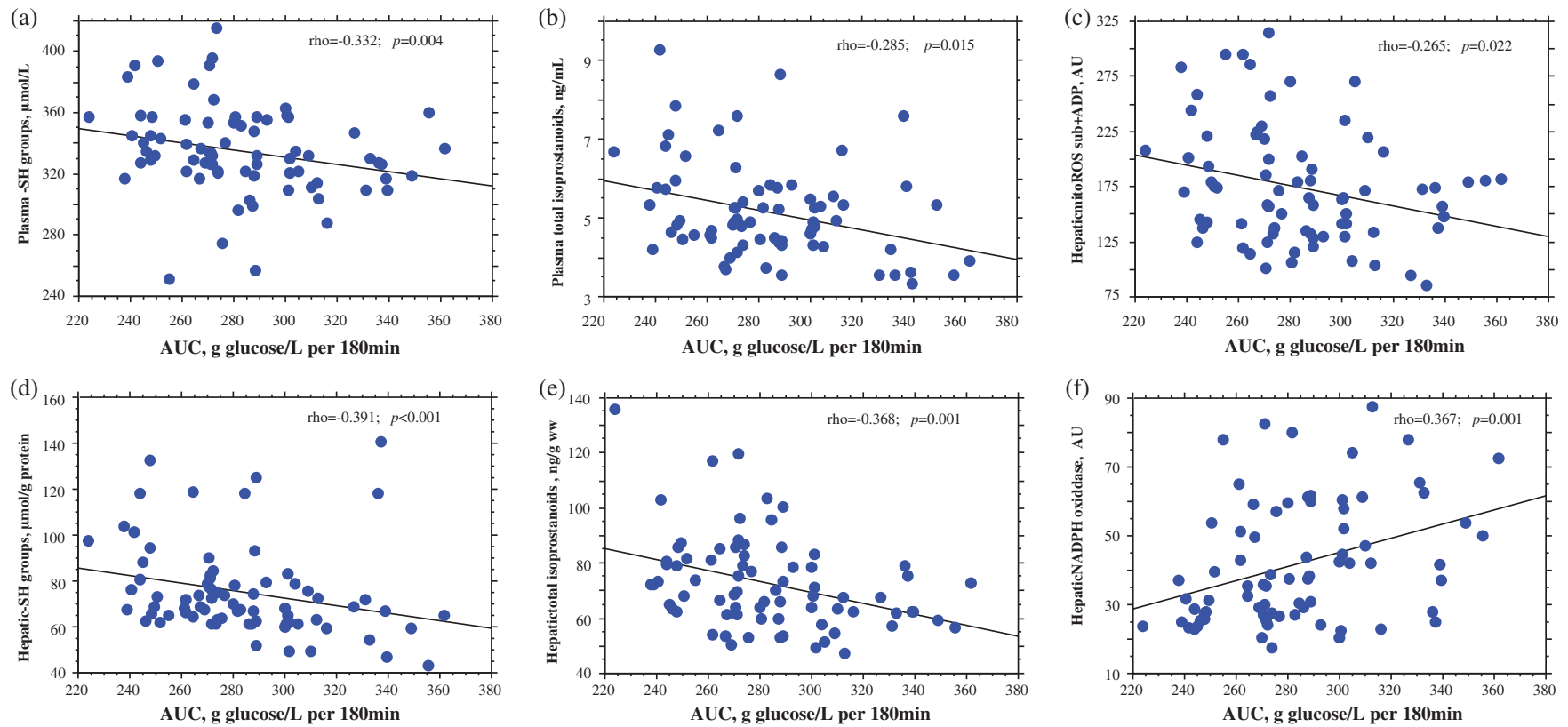

Fig. 9 Correlations between glucose tolerance test and major oxidative stress markersResults were expressed as means \pm SD, $n=6-8$ animals per group per diet treatment duration. Correlations were performed with the Spearman method. Glucose tolerance test was negatively correlated with some antioxidative stress parameters, namely plasma $-\mathrm{SH}$ groups (a), hepatic $-\mathrm{SH}$ groups (b), but also with plasma and hepatic isoprostanoids (c, d) and mitochondrial reactive oxygen species (ROS) production (e). Interestingly, it was positively correlated with hepatic NADPH oxidase-dependent superoxide radical production (f). AUC: area under the curve; - SH groups: sulfhydryl groups; and GSH: glutathione.

(Yang et al., 2016) has reported that oral administration of Salidroside prevented liver injury in the NASH model, through its antioxidant actions to suppress oxidative stress and the free radical-generating Nox2 in liver.

Several oxidative and antioxidative biomarkers have been proposed, including TBARS, hydroperoxides, protein sulfhydryl groups, and reduced and oxidized GSH as well as SOD, catalase, GPx to assess oxidative stress in vivo. However, isoprostanoids (such as $15 \mathrm{~F}_{2 \mathrm{t}}$-isoprostane) originated from PUFA and formed under autooxidative conditions are known to be excellent markers of lipid peroxidation in vivo and more generally of oxidative stress (Galano et al., 2017; Yin, 2008). Therefore, the strong point of this study is the measurement of not less than 14 isoprostanoid metabolites in both plasma and liver tissue. Surprisingly, the sum of isoprostanoid metabolites in both media was decreased with the HFHFr diet compared to the control diet. In addition, when the isoprostanoid metabolites were classified according to their fatty acid origin, the classes $\mathrm{B}, \mathrm{C}$, and $\mathrm{E}$ of plasma isoprostanoid metabolites were decreased, while the class $\mathrm{A}$ remained unchanged. More interestingly, four classes (A, B, C, and E) of liver isoprostanoid metabolites were decreased with the HFHFr diet compared to the control diet. Because the isoprostanoid metabolites are originated from specific PUFA, the ratio of isoprostanoid metabolites/corresponding PUFA was considered. Indeed, the liver PUFA percent was decreased by about $35 \%$ with the HFHFr diets compared to the control diet, but this does not explain alone the marked decrease in liver isoprostanoid metabolite contents as the liver lipid level and those of PUFA were much higher in the HFHFr rats (about $+100 \%$ ) compared to the control rats (Fouret et al., 2018). When these two factors are taken together, the liver total PUFA content decreased with the HFHFr diets compared to the control diet. However, the isoprostanoid metabolites/PUFA ratio remained significantly lower with the HFHFr diet compared to the control diet. To our knowledge, this is the first time that the isoprostanoid metabolites were reported in a high fat/high fructose diet-fed rat model in both plasma and liver tissue. In the majority of studies, only F2-isoprostane or 8-isoprostane was measured by ELISA in blood or urine and seldom in tissues, which gave often discordant results. These studies have reported either increased (Zhu et al., 2008) or unchanged or (Petersson et al., 2010) even decreased (Penumetcha et al., 2012) isoprostane levels with HF or HF/HS diets in human or rodent models.

In line with our isoprostanoid metabolites results, the plasma TBARS levels were also decreased whereas the liver TBARS levels remained unchanged. However, the other blood oxidative stress markers were only modestly modified, whereas those measured in the liver were highly affected with our HFHFr diet. Indeed, the protein oxidation assessed by the oxyblot kit showed that protein carbonylation was increased under the HFHFr diet since the early stages of the study. In addition, both liver sulfhydryl and 
GSH levels as well as the enzymatic activity of SOD, catalase, and GPx significantly decreased with the HFHFr diet compared to the control diet. This means that HFHFr diet induced marked oxidative stress of proteins but the oxidative stress of lipids was rather limited or inexistent.

In conclusion, in spite of long-term high-fat highfructose diet feeding and the high hepatic accumulation of lipids, including DAG and ceramides, the inflammation status as well as the ROS production and oxidative stress markers, in particular lipid peroxidation, that is isoprostanoids and TBARS, remained very low if any even after 20 weeks of HFHFr diet feeding. The mitochondrial ROS production remained unchanged while NADPH oxidase was increased with the HFHFr diet associated with some perturbation of hepatic protein peroxidation and its redox status. Most of these lipid and oxidative stress changes occurred at the same time and as early as 8 or 12 weeks of diet treatment. This implies that low-grade oxidative stress, which accompanied glucose intolerance and hepatic steatosis, may be the result, not the cause, of hepatic lipid accumulation, and suggests that marked hepatic oxidative stress should occur at the end of the steatotic stage to result in frank insulin resistance and steatohepatitis as well as other fatty liver diseases. These interesting findings need to be further evaluated in other animal species as well as in human studies.

Acknowledgements The authors wish to express their deep acknowledgements to Guillaume Reversat and Amandine Rocher for their technical aid in the isoprostanoid analysis. The authors wish also to thank Marie-Agnès Chauvin for completion of Oxyblot western blots and Charlène Couturier for qPCR analyses. The authors wish to acknowledge the significant contribution of the kind staff of the METAMUS DMEM facility, which belongs to Montpellier animal facilities (RAM, BIOCAMPUS).

Authors' Contributions Author contributions were as follows: study design (C.F.-C., C.C.), data collection (G.F., C.V., B.B., B.J., A.B.-Z., J.R., J.F.L.), statistical analysis (C.C. and C.F.-C.), data interpretation (C.F.-C., T.D., F.C., S.G., C.C.), manuscript preparation (C.F.-C., S.G., C.C.), literature search (C.F.-C. and S.G., C.C.) and funds collection (C.F.-C., S.G., C.C.).

Conflict of Interest The authors declare that they have no conflicts of interest.

\section{References}

Anderson, E. J., Lustig, M. E., Boyle, K. E., Woodlief, T. L., Kane, D. A., Lin, C. T., ... Neufer, P. D. (2009) Mitochondrial $\mathrm{H} 2 \mathrm{O} 2$ emission and cellular redox state link excess fat intake to insulin resistance in both rodents and humans. Journal of Clinical Investigation, 119:573-281.

Ando, K., \& Fujita, T. (2009) Metabolic syndrome and oxidative stress. Free Radical Biology and Medicine, 47:213-218.

Anvari, E., Wikstrom, P., Walum, E., \& Welsh, N. (2015) The novel NADPH oxidase 4 inhibitor GLX351322 counteracts glucose intolerance in high-fat diet-treated C57BL/6 mice. Free Radical Research, 49:1308-1318.

Aoun, M., Feillet-Coudray, C., Fouret, G., Chabi, B., Crouzier, D., Ferreri, C., ... Coudray, C. (2011) Rat liver mitochondrial membrane characteristics and mitochondrial functions are more profoundly altered by dietary lipid quantity than by dietary lipid quality: Effect of different nutritional lipid patterns. British Journal of Nutrition, 107:647-659.

Asrih, M., \& Jornayvaz, F. R. (2013) Inflammation as a potential link between nonalcoholic fatty liver disease and insulin resistance. Journal of Endocrinology, 218:R25-R36.

Barja, G. (2002) The quantitative measurement of $\mathrm{H} 2 \mathrm{O} 2$ generation in isolated mitochondria. Journal of Bioenergetics and Biomembranes, 34:227-233.

Bedard, K., \& Krause, K. H. (2007) The NOX family of ROSgenerating NADPH oxidases: Physiology and pathophysiology. Physiological Reviews, 87:245-313.

Beers, R., \& Sizer, I. (1952) A spectrophotometric method for measuring the breakdown of hydrogen peroxide by catalase. Journal of Biological Chemistry, 195:133-140.

Blachnio-Zabielska, A. U., Persson, X. M., Koutsari, C., Zabielski, P., \& Jensen, M. D. (2012) A liquid chromatography/tandem mass spectrometry method for measuring the in vivo incorporation of plasma free fatty acids into intramyocellular ceramides in humans. Rapid Communications in Mass Spectrometry, 26: 1134-1140.

Blachnio-Zabielska, A. U., Zabielski, P., \& Jensen, M. D. (2013) Intramyocellular diacylglycerol concentrations and [U-(1)(3)C]palmitate isotopic enrichment measured by LC/MS/MS. Journal of Lipid Research, 54:1705-1711.

Bradford, M. M. (1976) A rapid and sensitive method for the quantitation of microgram quantities of protein utilizing the principle of protein-dye binding. Analytical Biochemistry, 72:248-254.

Brandes, R. P., Weissmann, N., \& Schroder, K. (2014) Nox family NADPH oxidases: Molecular mechanisms of activation. Free Radical Biololy and Medicine, 76:208-226.

Bravard, A., Bonnard, C., Durand, A., Chauvin, M. A., Favier, R., Vidal, H., \& Rieusset, J. (2011) Inhibition of xanthine oxidase reduces hyperglycemia-induced oxidative stress and improves mitochondrial alterations in skeletal muscle of diabetic mice. American Journal of Physiology-Endocrinology and Metabolism, 300: E581-E591.

Cardoso, A. R., Cabral-Costa, J. V., \& Kowaltowski, A. J. (2010) Effects of a high fat diet on liver mitochondria: Increased ATPsensitive $\mathrm{K}+$ channel activity and reactive oxygen species generation. Journal of Bioenergetics and Biomembranes, 42:245-253.

Dandona, P., Aljada, A., Chaudhuri, A., Mohanty, P., \& Garg, R. (2005) Metabolic syndrome: A comprehensive perspective based on interactions between obesity, diabetes, and inflammation. Circulation, 111:1448-1454.

Dupuy, A., Le Faouder, P., Vigor, C., Oger, C., Galano, J. M., Dray, C., ... Bertrand-Michel, J. (2016) Simultaneous quantitative profiling of 20 isoprostanoids from omega-3 and omega- 6 polyunsaturated fatty acids by LC-MS/MS in various biological samples. Analytica Chimica Acta, 921:46-58.

Feillet-Coudray, C., Sutra, T., Fouret, G., Ramos, J., WrutniakCabello, C., Cabello, G., ... Coudray, C. (2009) Oxidative stress in rats fed a high-fat high-sucrose diet and preventive effect of polyphenols: Involvement of mitochondrial and $\mathrm{NAD}(\mathrm{P}) \mathrm{H}$ oxidase systems. Free Radical Biology and Medicine, 46:624-632.

Fernandez-Sanchez, A., Madrigal-Santillan, E., Bautista, M., Esquivel-Soto, J., Morales-Gonzalez, A., Esquivel-Chirino, C., ... Morales-Gonzalez, J. A. (2011) Inflammation, oxidative stress, and obesity. International Journal of Molecular Sciences, 12: 3117-3132. 
Finck, B. N., \& Hall, A. M. (2015) Does diacylglycerol accumulation in fatty liver disease cause hepatic insulin resistance? BioMed Research International, 2015:104132.

Flohe, L., \& Gunzler, W. A. (1984) Assays of glutathione peroxidase. Methods in Enzymology, 105:114-121.

Fouret, G., Gaillet, S., Lecomte, J., Bonafos, B., Djohan, F., Barea, B., ... Feillet-Coudray, C. (2018) 20-week follow-up of hepatic steatosis installation and liver mitochondrial structure and activity and their interrelation in rats fed a high-fat-high-fructose diet. British Journal of Nutrition, 1019:368-380.

Furukawa, S., Fujita, T., Shimabukuro, M., Iwaki, M., Yamada, Y., Nakajima, Y., ... Shimomura, I. (2004) Increased oxidative stress in obesity and its impact on metabolic syndrome. Journal of Clinical Investigation, 114:1752-1761.

Galano, J. M., Lee, Y. Y., Oger, C., Vigor, C., Vercauteren, J., Durand, T., ... Lee, J. C. (2017) Isoprostanes, neuroprostanes and phytoprostanes: An overview of 25years of research in chemistry and biology. Progress in Lipid Research, 68:83-108.

Gerner, R. R., Wieser, V., Moschen, A. R., \& Tilg, H. (2013) Metabolic inflammation: Role of cytokines in the crosstalk between adipose tissue and liver. Canadian Journal of Physiology and Pharmacology, 91:867-872.

Griffith, O. W. (1980) Determination of glutathione and glutathione disulfide using glutathione reductase and 2-vinylpyridine. Analytical Biochemistry, 106:207-212.

Jocelyn, P. C. (1987) Spectrophotometric assay of thiols. Methods in Enzymology, 143:44-67.

Johnson, R. J., Stenvinkel, P., Martin, S. L., Jani, A., SanchezLozada, L. G., Hill, J. O., \& Lanaspa, M. A. (2013) Redefining metabolic syndrome as a fat storage condition based on studies of comparative physiology. Obesity (Silver Spring), 21:659-664.

Kawano, Y., \& Cohen, D. E. (2013) Mechanisms of hepatic triglyceride accumulation in non-alcoholic fatty liver disease. Journal of Gastroenterology, 48:434-441.

Li, S., Hong, M., Tan, H. Y., Wang, N., \& Feng, Y. (2016) Insights into the role and interdependence of oxidative stress and inflammation in liver diseases. Oxidative Medicine and Cellular Longevity, 2016:4234061.

Mantena, S. K., King, A. L., Andringa, K. K., Eccleston, H. B., \& Bailey, S. M. (2008) Mitochondrial dysfunction and oxidative stress in the pathogenesis of alcohol- and obesity-induced fatty liver diseases. Free Radical in Biology and Medicine, 44:1259-1272.

Marklund, S. (1976) Spectrophotometric study of spontaneous disproportionation of superoxide anion radical and sensitive direct assay for superoxide dismutase. Journal of Biological Chemistry, 251: 7504-7507.

Nakamura, A., \& Terauchi, Y. (2013) Lessons from mouse models of high-fat diet-induced NAFLD. International Journal of Molecular Sciences, 14:21240-21257.

Paik, Y. H., \& Brenner, D. A. (2011) NADPH oxidase mediated oxidative stress in hepatic fibrogenesis. Korean Journal of Hepatology, 17:251-257.

Peng, Y., Rideout, D., Rakita, S., Lee, J., \& Murr, M. (2012) Dietinduced obesity associated with steatosis, oxidative stress, and inflammation in liver. Surgery for Obesity and Related Diseases, $\mathbf{8}$ : 73-81.

Penumetcha, M., Song, M., Merchant, N., \& Parthasarathy, S. (2012) Pretreatment with n-6 PUFA protects against subsequent high fat diet induced atherosclerosis-potential role of oxidative stressinduced antioxidant defense. Atherosclerosis, 220:53-58.
Perry, R. J., Samuel, V. T., Petersen, K. F., \& Shulman, G. I. (2014) The role of hepatic lipids in hepatic insulin resistance and type 2 diabetes. Nature, 510:84-91.

Petersen, M. C., \& Shulman, G. I. (2017) Roles of diacylglycerols and ceramides in hepatic insulin resistance. Trends in Pharmacological Sciences, 38:649-665.

Petersson, H., Riserus, U., McMonagle, J., Gulseth, H. L., Tierney, A. C., Morange, S., ... Basu, S. (2010) Effects of dietary fat modification on oxidative stress and inflammatory markers in the LIPGENE study. British Journal of Nutrition, 104:1357-1362.

Renaud, H. J., Cui, J. Y., Lu, H., \& Klaassen, C. D. (2014) Effect of diet on expression of genes involved in lipid metabolism, oxidative stress, and inflammation in mouse liver-insights into mechanisms of hepatic steatosis. PLoS One, 9:e88584.

Roberts, C. K., Barnard, R. J., Sindhu, R. K., Jurczak, M., Ehdaie, A., \& Vaziri, N. D. (2006) Oxidative stress and dysregulation of NAD $(\mathrm{P}) \mathrm{H}$ oxidase and antioxidant enzymes in diet-induced metabolic syndrome. Metabolism, 55:928-934.

Sunderman Jr., F. W., Marzouk, A., Hopfer, S. M., Zaharia, O., \& Reid, M. C. (1985) Increased lipid peroxidation in tissues of nickel chloride-treated rats. Annals of Clinical \& Laboratory Science, 15: 229-236.

Tilg, H., \& Moschen, A. R. (2010) Evolution of inflammation in nonalcoholic fatty liver disease: The multiple parallel hits hypothesis. Hepatology, 52:1836-1846.

Tordjman, J., Guerre-Millo, M., \& Clement, K. (2008) Adipose tissue inflammation and liver pathology in human obesity. Diabetes \& Metabolism, 34:658-663.

Ucar, F., Sezer, S., Erdogan, S., Akyol, S., Armutcu, F., \& Akyol, O. (2013) The relationship between oxidative stress and nonalcoholic fatty liver disease: Its effects on the development of nonalcoholic steatohepatitis. Redox Report, 18:127-133.

Valko, M., Leibfritz, D., Moncol, J., Cronin, M. T., Mazur, M., \& Telser, J. (2007) Free radicals and antioxidants in normal physiological functions and human disease. International Journal of Biochemistry \& Cell Biology, 39:44-84.

Vial, G., Dubouchaud, H., Couturier, K., Cottet-Rousselle, C., Taleux, N., Athias, A., ... Leverve, X. M. (2011) Effects of a highfat diet on energy metabolism and ROS production in rat liver. Journal of Hepatology, 54:348-356.

Witko-Sarsat, V., Friedlander, M., Nguyen Khoa, T., CapeillereBlandin, C., Nguyen, A. T., Canteloup, S., ... DescampsLatscha, B. (1998) Advanced oxidation protein products as novel mediators of inflammation and monocyte activation in chronic renal failure. Journal of Immunology, 161:2524-2532.

Yagi, K. (1984) Assay for blood plasma or serum. Methods in Enzymology, 105:328-331.

Yang, Z. R., Wang, H. F., Zuo, T. C., Guan, L. L., \& Dai, N. (2016) Salidroside alleviates oxidative stress in the liver with nonalcoholic steatohepatitis in rats. BMC Pharmacology and Toxicology, 17:16.

Yin, H. (2008) New techniques to detect oxidative stress markers: Mass spectrometry-based methods to detect isoprostanes as the gold standard for oxidative stress in vivo. BioFactors, 34:109-124.

Zhu, M. J., Sun, L. J., Liu, Y. Q., Feng, Y. L., Tong, H. T., Hu, Y. H., \& Zhao, Z. (2008) Blood F2-isoprostanes are significantly associated with abnormalities of lipid status in rats with steatosis. World Journal of Gastroenterology, 14:4677-4683.

Zivkovic, A. M., German, J. B., \& Sanyal, A. J. (2007) Comparative review of diets for the metabolic syndrome: Implications for nonalcoholic fatty liver disease. American Journal of Clinical Nutrition, 86:285-300. 